ARTICLE

\title{
Intracellular Staphylococcus aureus persisters upon antibiotic exposure
}

Frédéric Peyrusson", Hugo Varet (10 2 ${ }^{2}$, Tiep Khac Nguyen', Rachel Legendre (1) 2, Odile Sismeiro ${ }^{3}$, Jean-Yves Coppée ${ }^{3}$, Christiane Wolz (i) ${ }^{4}$, Tanel Tenson ${ }^{5}$ \& Françoise Van Bambeke (D) ${ }^{1 \times}$

Bacterial persister cells are phenotypic variants that exhibit a transient non-growing state and antibiotic tolerance. Here, we provide in vitro evidence of Staphylococcus aureus persisters within infected host cells. We show that the bacteria surviving antibiotic treatment within host cells are persisters, displaying biphasic killing and reaching a uniformly non-responsive, non-dividing state when followed at the single-cell level. This phenotype is stable but reversible upon antibiotic removal. Intracellular S. aureus persisters remain metabolically active but display an altered transcriptomic profile consistent with activation of stress responses, including the stringent response as well as cell wall stress, SOS and heat shock responses. These changes are associated with multidrug tolerance after exposure to a single antibiotic. We hypothesize that intracellular S. aureus persisters may constitute a reservoir for relapsing infection and could contribute to therapeutic failures.

\footnotetext{
${ }^{1}$ Pharmacologie cellulaire et moléculaire, Louvain Drug Research Institute, Université catholique de Louvain (UCLouvain), Brussels, Belgium. ${ }^{2}$ Hub de Bioinformatique et Biostatistique - Département Biologie Computationnelle, Institut Pasteur, USR 3756 CNRS, Paris, France. ${ }^{3}$ Institut Pasteur, Plate-forme Transcriptome et Epigenome, Biomics, Centre de Ressources et Recherches Technologiques (C2RT), Paris, France. ${ }^{4}$ Institut für Medizinische Mikrobiologie

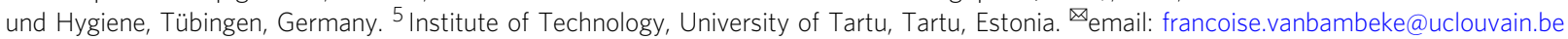


ersisters are subpopulations of cells in bacterial cultures that adopt a transient phenotype characterized by a nongrowing state and a tolerance to lethal concentrations of antibiotics ${ }^{1}$. As opposed to resistance, persistence is not genetically inherited. Experimentally, persisters are usually evidenced by biphasic kill curves, when a bulk of susceptible bacteria is rapidly killed by exposure to high antibiotic concentrations, while a small proportion survives for longer time ${ }^{2,3}$. Recent advances in single-cell analyses have allowed characterizing persisters in more details, and notably revealed their non-growing state. There is now convincing and convergent experimental evidence of their clinical relevance, as they contribute to the establishment of chronic infections as well as to the emergence of antibiotic resistance ${ }^{4}$.

The switch to persister phenotype has been largely related to the activation of the stringent response, a global and widely distributed adaptation program that occurs in response to various stresses and modulates many physiological activities. However, its exact role in persistence regulation is still debated ${ }^{5,6}$. Additionally, tolerance is often considered as a passive consequence of growth arrest ${ }^{7}$, which is now challenged by mounting evidence of active responses in some species ${ }^{8}$. Similarly, whether or not dormancy is sufficient to explain antibiotic tolerance is also questioned ${ }^{9}$.

Although persister cells have been identified in all major pathogens ${ }^{10-13}$, a switch to a persister phenotype has been proposed to occur inside eukaryotic cells for only very few intracellular bacteria ${ }^{14,15}$ in response to the environmental stress imposed by the host cell.

Intracellular survival of Staphylococcus aureus is widely recognized as a major factor in the recurrence of infections ${ }^{16}$ and intracellular forms of $S$. aureus have been shown to become less responsive to antibiotic action ${ }^{17}$, suggesting a switch to a persister phenotype.

In the present work, we provide evidence for the presence of $S$. aureus intracellular persisters after antibiotic exposure and characterize their dynamics using a fluorescence dilution-based method to monitor bacterial division at the single cell level. We show that intracellular bacterial populations are characterized by a biphasic killing, accompanied by a rapid switch to a uniformly non-dividing and non-responsive state, which is readily reversible upon antibiotic removal. As a potential issue in therapeutic failures, we then aim to better understand the factors leading to antibiotic persistence and tolerance. Using RNA-sequencing we show that these persisters harbor a major transcriptomic reprogramming and remain metabolically active despite prolonged persistence within the host cells. While neither ATP nor amino acid limitation occur, we find that bacteria adjust their central carbon metabolism and redirect transcription to the benefit of a network of adaptive responses. Strikingly, after exposure to a single antibiotic, these responses lead to tolerance to multiple antibiotic classes that act on unrelated targets.

\section{Results}

S. aureus surviving to antibiotics in cells are persisters. Concentration-response curves of typical antistaphylococcal antibiotics targeting the cell wall (oxacillin), protein synthesis (clarithromycin), and replication (moxifloxacin), revealed their inability to clear bacteria from J774 macrophages: after $24 \mathrm{~h}$ of infection with high antibiotic concentrations, an antibiotictolerant pool of cultivable $S$. aureus persisted inside the macrophages (Fig. 1a). In parallel, time-kill curves performed in the presence of high concentration of each of these antibiotics revealed a biphasic killing: a bulk of the bacterial population was susceptible and rapidly killed while a subpopulation with a slower killing rate was persisting for a much longer period of time. A similar profile was observed against planktonic cultures, but the persisting subpopulation was considerably lower than intracellularly (Fig. 1b). This profile is considered as a hallmark of antibiotic persistence ${ }^{2,3}$.

Persisters are subpopulations that transiently adopt a nongrowing and a tolerant state. To confirm these observations and further characterize this phenotype, we first set out to provide evidence for non-growing phenotype of intracellular $S$. aureus by setting up a fluorescence dilution-based method to monitor bacterial division at the single cell level ${ }^{18}$. We used $S$. aureus SH1000 expressing GFP from a tetracycline-inducible promotor, which allows one to follow cell division by monitoring the decrease in GFP signal intensity per cell after removal of the inducer. To validate this approach, bacteria in broth were induced overnight for GFP production, washed from inducer, and diluted to entry into exponential phase, after which their fluorescence signals were analyzed by flow cytometry (Fig. 1c). A homogeneous replication within the bacterial population was observed with unimodal distribution of the signal gradually declining over time, as confirmed in microscopy (Fig. 1d). When comparing the fluorescence dilution with the cfu counting, both methods revealed similar growth curves for 5 generations and similar doubling times (Fig. 1e), validating fluorescence dilution for measurement of bacterial replication ${ }^{19}$.

The same method was applied to characterize the dynamics of intracellular S. aureus replication. Macrophages infected by GFPexpressing inoculum revealed bacteria with apparent normal morphology and distinct fluorescence status depending on the antibiotic pressure (Fig. 1f).

This was examined by analyzing the flow cytometry profiles of the replication of intraphagocytic bacteria challenged with different antibiotic concentrations during $48 \mathrm{~h}$ of infection (Fig. 1g). Exposure to low antibiotic pressure resulted in an equilibrium between killing and replication. Among the bulk of growing bacteria, a subpopulation rapidly entered into a nongrowing state (Supplementary Fig. 1a, b). High antibiotic pressure resulted in both killing of replicating bacteria and larger amounts of non-growing bacteria, leaving a homogenous population of non-growing persisters until the end of the experiment.

Persisters revert to a normal phenotype once antibiotic pressure is removed. We therefore determined the reversibility of the phenotype after antibiotic removal. To this effect, we tested growth resumption and susceptibility towards antibiotics within a non-replicating population collected by cell sorting from infected macrophages after reinoculation in broth (Fig. 1h). A full reversion of the phenotype was observed, in terms of both resulting growth at $24 \mathrm{~h}$ and antibiotic susceptibility. Additionally, a biphasic killing profile was also observed for bacteria harvested from macrophages (Fig. 1i). This transient bidirectional switch demonstrates that these intracellular non-dividing subpopulations are persisters. Reversion was also confirmed intracellularly, where persisters started dividing spontaneously within the cell after removal of the antibiotic pressure (Fig. $1 \mathrm{j}$ and Supplementary Fig. 1c, d).

This intracellular persistence, together with the ability to resume intracellular replication, are considered as two key determinants for relapsing infections and likely contribute to the clinical observation of rapid recolonization soon after the end of therapy ${ }^{20}$. Intracellular replication is largely described in nonprofessional phagocytes, while intracellular persistence has been reported, although not systematically, in professional phagocytes $^{21}$, and referred to as cell induced-persistence ${ }^{22}$. Yet, intrinsic cell defense mechanisms are critical to trigger either persistence or replication. Illustrating this duality, we observed active bacterial replication in untreated J774 macrophages but not in primary human macrophages (Fig. $1 \mathrm{k}, 1$ and Supplementary 
a

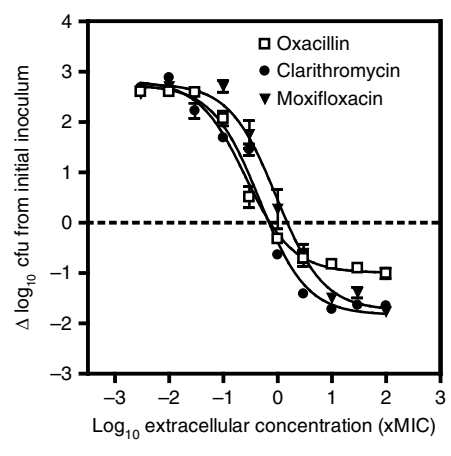

d

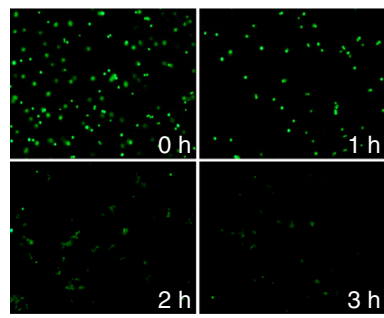

$\mathbf{g}_{100}$
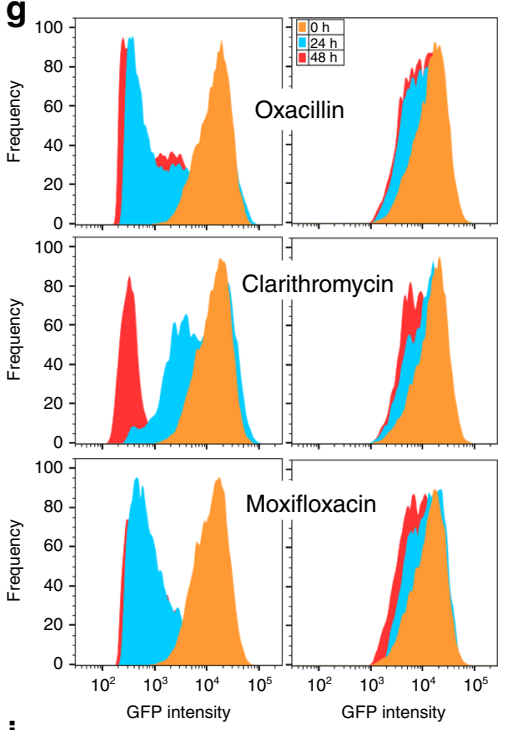

j

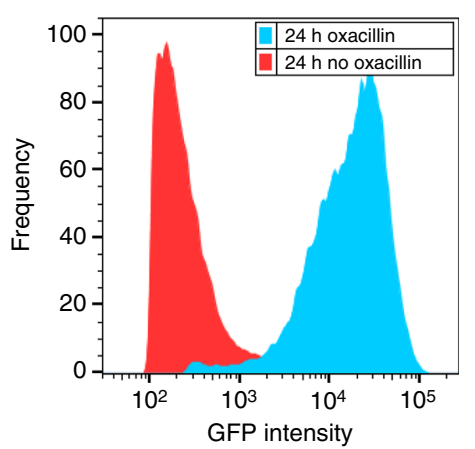

b

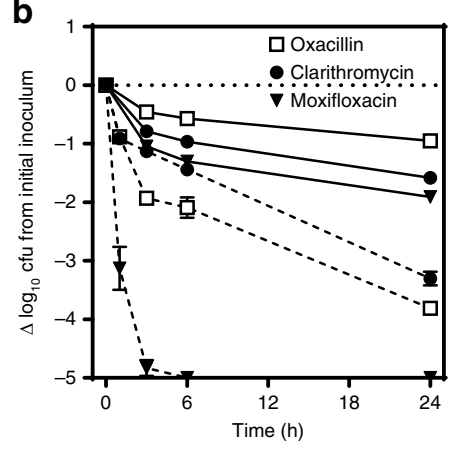

e

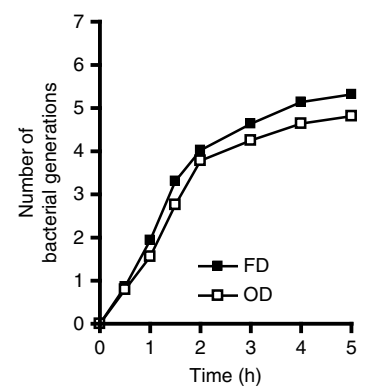

h

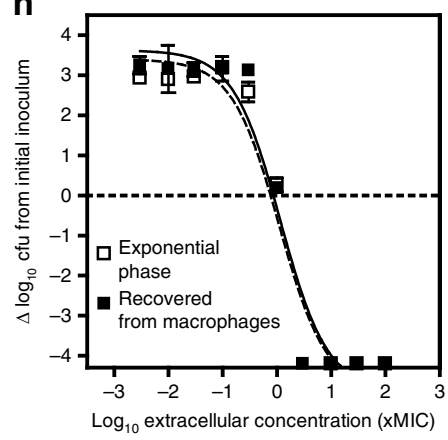

k

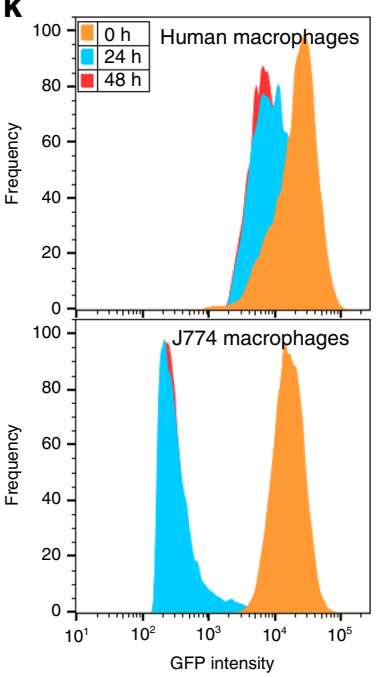

C

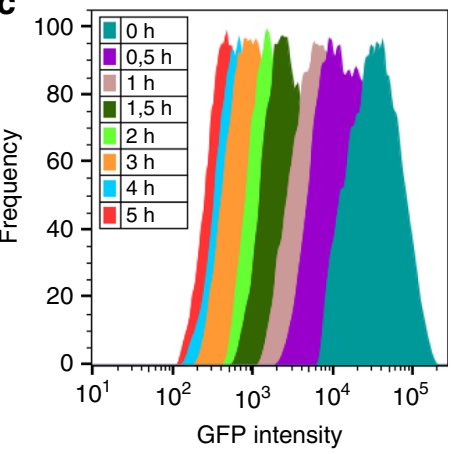

f

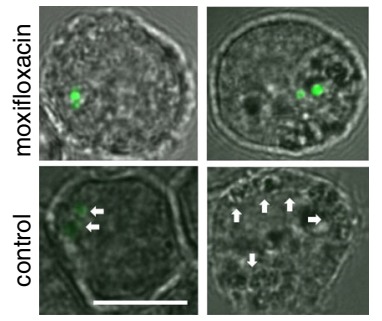

i

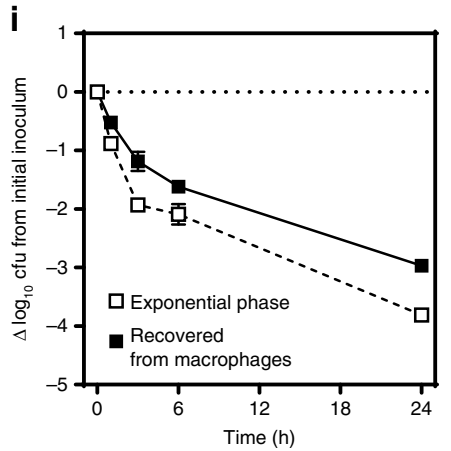

I

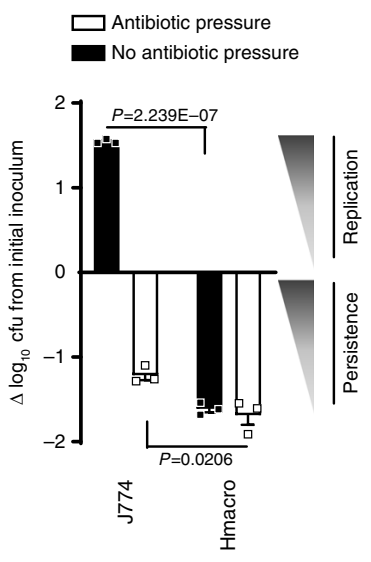

Fig. 1e, f), which can host a viable persister pool, albeit less abundant than the one induced by antibiotic pressure in J774 macrophages.

Thus, we propose here a model in which antibiotic pressure could represent a major trigger factor for intracellular persistence in more permissive cell types. Similar experiments were performed in a series of human cells (epithelial cells, monocytes, osteoblasts, keratinocytes) and confirm the general character of these observations (Supplementary Fig. 2). Because these antibiotic-induced persisters readily reverse upon drug removal, they could constitute a viable reservoir that acts as a major source of dissemination and relapsing infections.

To better understand the factors underlying persistence, we undertook an in-depth RNA-sequencing analysis of these 
Fig. 1 Evidence and dynamics of intracellular persisters of S. aureus. a Antibiotic activity against S. aureus infecting $\mathbf{J 7 7 4}$ macrophages exposed to increasing concentrations of antibiotics for $24 \mathrm{~h}$ (data expressed as $\log _{10}$ cfu reduction from postphagocytosis inoculum). $\mathbf{b}$ Time-kill curves against $\mathrm{S}$. aureus infecting $\mathbf{5 7 7 4}$ macrophages (solid lines) or in exponential phase culture (dotted lines) exposed to 50x MIC of antibiotics for the indicated periods. c Fluorescence dilution (FD) experiment with S. aureus expressing inducible GFP. Bacteria washed from inducer at the entry of exponential phase were grown in fresh broth. The graph shows flow cytometric profiles of the frequency of events as a function of GFP intensity over time. $\mathbf{d}$ Corresponding images in epifluorescence microscopy. e Corresponding bacterial replication curves determined by FD and $\mathrm{OD}_{620 \mathrm{~nm}}$ (OD), which displayed similar doubling times (e.g., $27 \mathrm{~min}$ and $28.7 \mathrm{~min}$ between $1 \mathrm{~h}$ and $2 \mathrm{~h}$, respectively; $N$ [number of generations]). $\mathbf{f}$ Confocal microscopy of infected J774 macrophages exposed to 50x MIC moxifloxacin or under control conditions ( $2 x$ MIC gentamicin) for $24 \mathrm{~h}$. Arrows: bacteria with diluted signal (bar: $10 \mu \mathrm{m}$ ). $\mathbf{g}$ Flow cytometric profiles of bacteria recovered from macrophages exposed to $2 \times$ (left) or $50 x$ MIC (right) of each antibiotic for the indicated periods. $\mathbf{h}$, $\mathbf{i}$ Activity of oxacillin (h, concentration-effect at $24 \mathrm{~h}$; i, time-kill curve with $50 x$ MIC oxacillin) in broth, against an exponential phase culture (open symbols) or bacteria recovered from macrophages exposed to 50x MIC oxacillin for $24 \mathrm{~h}$ (closed symbols). $\mathbf{j}$ Flow cytometric profiles of bacteria recovered from macrophages exposed to 50x MIC oxacillin for $24 \mathrm{~h}$ (blue), then washed from oxacillin and reincubated in control conditions ( $2 \times$ MIC gentamicin) for an additional period of $24 \mathrm{~h}$ (red). $\mathbf{k}$ Flow cytometric profiles of bacteria recovered from control (2x MIC gentamicin) $\mathrm{J} 774$ and human macrophages for the indicated periods. I Intracellular inoculum in infected $\mathrm{J774}$ and human macrophages incubated for $24 \mathrm{~h}$ with $50 \times$ MIC oxacillin or in control conditions (2x MIC gentamicin). Statistical significance was determined by two-tailed Student's t-test. Data are means \pm SEM $(\mathbf{a}, \mathbf{b}, \mathbf{h}, \mathbf{i}, \mathbf{l})$ or representatives results $(\mathbf{c}, \mathbf{d}, \mathbf{e}, \mathbf{f}, \mathbf{g}, \mathbf{j}, \mathbf{k})$ of three independent experiments. $\mathbf{a}, \mathbf{b}, \mathbf{e}, \mathbf{h}, \mathbf{i}, \mathbf{l}$, Source data are provided as a Source Data file.

intracellular persisters induced by prolonged antibiotic exposure within eukaryotic cells, as a model for environmental stresses that bacteria face in a clinical context.

Persisters exhibit an altered transcriptomic profile. Macrophages were infected by GFP-expressing bacteria and challenged by oxacillin for $24 \mathrm{~h}$ to induce homogeneous populations of persisters. Cell sorting was used to isolate the subset of GFPexpressing bacteria $(\mathrm{GFP}+)$ that display a propidium iodide negative signal (PI-) (Fig. 2a). The vast majority of GFP+/PIevents were able to form colonies, confirming that they were viable.

The transcriptomic profile of sorted persisters was assessed by RNA-sequencing and reads of each sample were mapped on 2967 protein-coding genes from the reference genome ${ }^{23}$. Differential expression analysis identified 1477 differentially expressed genes (DEG) between the intracellular persisters and control bacteria (Fig. 2b). Hierarchical clustering of this sample set revealed both major divergences between these conditions and high withingroup reproducibility. Of the 1477 DEGs, 710 were upregulated and 767 downregulated in persisters (Supplementary Fig. 3).

Over-representation analysis of DEGs revealed that the vast majority of significantly enriched functions corresponds to enrichments in downregulated genes (Fig. 2c). A large group of those belongs to metabolism processes, indicating an overall decrease in metabolic activities, some of them being typically associated with proliferation processes (e.g., nucleotide metabolism and oxidative phosphorylation). Of interest, proliferationrelated genes are described to be repressed to the benefit of genes required for stress-defense mechanisms in persisters ${ }^{24}$. Downregulated gene-sets also unveil an important enrichment in metabolism of amino acids (e.g., valine, leucine, isoleucine and lysine biosynthesis), as well as in aminoacyl-tRNA synthetases. Conversely, regarding enrichment in upregulated functions, galactose metabolism was the most significantly enriched function, which may point to deep metabolic network alterations.

Stringent response contributes to the persistence switch. The persister phenotype has been largely related to the activation of the stringent response $(\mathrm{SR})^{25}$. In response to diverse stresses (including starvation signals and antibiotics), SR is mediated by the rapid synthesis of the alarmones (p)ppGpp, leading to deep transcriptomic reprogramming, especially the repression of proliferation-related genes and the activation of stress resistanceand starvation survival-related genes and a halt of bacterial division ${ }^{24}$. However, recent reports challenge its exact role as a central regulator of persistence 26,27 .

In most firmicutes, (p)ppGpp is synthesized from the GDP/ GTP pool via three enzymes: the bifunctional enzyme Rsh that possesses synthase and hydrolase domains, and RelP and RelQ, which only have a synthase domain ${ }^{24}$. The molecular responses initiated by (p)ppGpp seem to differ among species. In some firmicutes, (p)ppGpp has been proposed to affect transcription indirectly through a reduction in the intracellular pool of nucleotides following (p)ppGpp synthesis ${ }^{28}$. Interestingly, the expression of regulators differs depending on the nature of the stress: while Rsh is mainly induced under amino acid limitation ${ }^{29}$, RelP and RelQ have been shown to be mostly induced by cell wall-targeting antimicrobials ${ }^{30}$. In S. aureus, CodY regulon is also an integral part of SR: under amino acid starvation, silenced genes are mainly downregulated through the inhibitory effect of $(p)$ ppGpp whereas the majority of activated genes are indirectly regulated via de-repression of CodY. Yet, the implication of SR in intracellular persistence has not yet been conclusively clarified.

Because SR is a highly dynamic process, we investigated the expression of its regulatory network during infection. Quantitative RT-PCR indicated a rapid and transitory boost of these regulators soon after uptake of $S$. aureus by macrophages (Fig. 3a): relQ reached its maximal transcription level $30 \mathrm{~min}$ after phagocytosis, and $r e l P, \operatorname{cod} Y$, and, to a lesser extent $r s h$, after 2 h. Limited expression of $r s h$ is compatible with its pivotal role in the SR: due to its dual hydrolase/synthase activity, Rsh finely balances the basal levels of (p)ppGpp and prevents its toxic accumulation $^{31}$.

Conversely, at later time points, our transcriptomic approach indicates that the vast majority $(73 \%)$ of genes within the SR stimulon display a divergent expression signature (repression or non-statistically significant changes; Supplementary Fig. 4), further supporting that the activation of SR is transitory.

To delineate the contribution of SR to the observed phenotype, macrophages were infected with mutants defective in the rsh synthase domain and/or codY (HG001 strain and isogenic mutants; see Supplementary Table 1) and exposed to high antibiotic pressure (Fig. 3b). Interestingly, with all antibiotics tested, the load of persisters was lower in the rsh-negative background than in the parental strain, the difference being less marked with oxacillin. Double mutation in SR regulators led to an additional decrease in the residual load of persisters with oxacillin, and reached maximal effect upon moxifloxacin exposure.

These results strongly suggest that SR is transiently implicated during the initiation of intracellular persistence through $(\mathrm{p})$ 
a
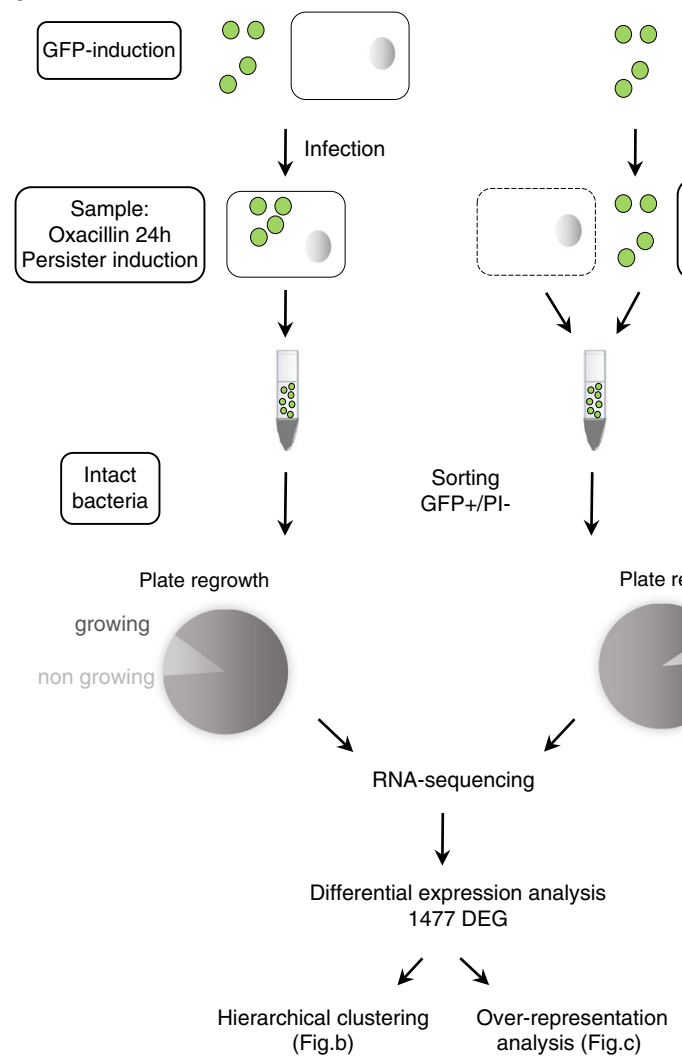
(Fig.b)
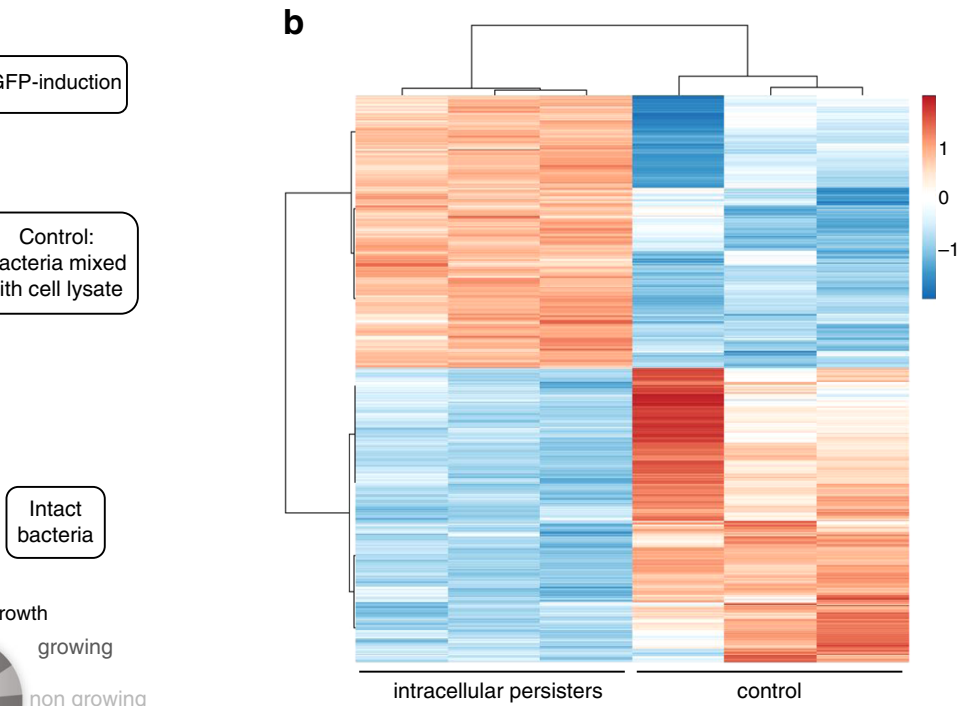

with cell lysate

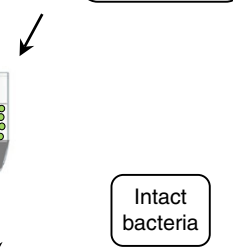

Plate regrowth

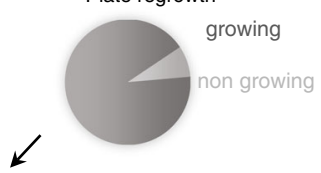

intracellular persisters

control

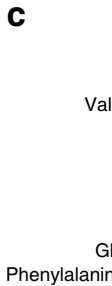
Glycine, serine and threonine metabolism (30) -
Phenylalanine, tyrosine and tryptophan biosynthesis (19) -

Vitamin B6 metabolism (4) Monobactam biosynthesis (5) Carotenoid biosynthesis (5) Valine, leucine and isoleucine biosynthesis (13) Lysine biosynthesis (12) Nitrogen metabolism (12) Nitrogen metabolism (12) - 1 Methane metabolism (18) One carbon pool by folate (10) Galactose metabolism (20) Aminoacyl-tRNA biosynthesis (24) Glycolysis / Gluconeogenesis (39) 2-Oxocarboxylic acid metabolism (20) Biosymthesis of amino acids (101) Cysteine and methionine metabolism (23) Glyoxylate and dicarboxylate metabolism (20) Purine metabolism (44) Biosynthesis of antibiotics (168) Biosynthesis of secondary metabolites (226) Microbial metabolism in diverse environments (133) Metabolic pathways (499) Carbon metabolism (81)

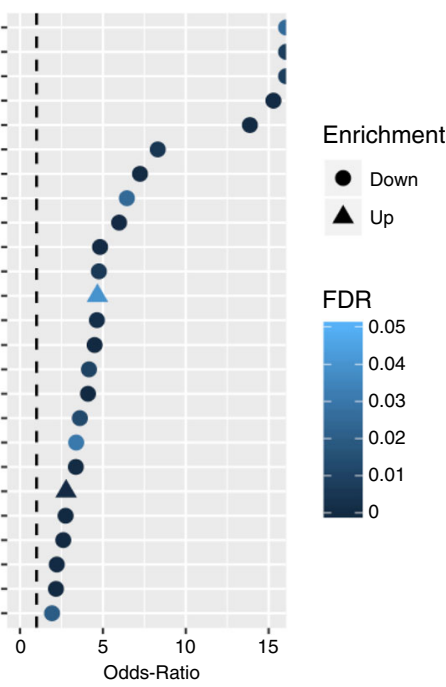

Fig. 2 Intracellular antibiotic-induced persisters exhibit a profoundly altered transcriptomic profile. a Experimental procedure for sorting and RNAsequencing of S. aureus persisters of SH1000. Cells infected by GFP-expressing bacteria were exposed to 50x MIC oxacillin to allow for the induction of a homogeneous population of persisters. The subset of intact persisters and control samples were collected and sorted by fluorescence-activated cell sorting (FACS) by gating GFP positive (GFP+) and propidium iodide negative signals (PI-), and processed for RNA sequencing (three replicates per condition). $89 \%$ and $92 \%$ of sorted bacteria from samples and control were able to form colonies, respectively. Differentially expressed genes (DEG) were then analyzed by hierarchical clustering and over-representation analysis. b Heatmap displaying hierarchical clustering of DEGs between intracellular persisters and control samples from three biological replicates (color code is function of a Variance Stabilizing Transformation [VST]). c Over-representation analysis of DEGs. The graph displays over-represented up- and downregulated KEGG gene-sets (EnrichmentBrowser R package), using the Fisher's Exact Test and evaluated through Odds-Ratio. Only gene-sets with a false discovery rate (FDR) lower than 0.05 were considered significantly enriched. Numbers in brackets represent the number of genes in the gene-set.

ppGpp regulations, in a variable manner depending on the drug exposure. This supports the hypothesis that the central regulation is not entirely dependent on SR and that multiple pathways contribute to the persister phenotype.

Persisters display dysregulated but active protein synthesis. Originally, persisters were described as being in a strictly dormant state, in which SR shuts down energy-consuming processes by turning translation off, thereby leading to antibiotic tolerance ${ }^{32}$.

In our study, the transcriptomic profile of translation-related genes reveals a dysregulated pattern (Fig. 3c). Indeed, typical members of the protein synthesis machinery were found activated, and displayed a similar trend throughout the whole duration of infection (Supplementary Fig. 5). In line with enrichment analysis, aminoacyl-tRNA synthetase encoding genes were, by contrast, deeply silenced, as is typically encountered under stringent conditions. Additionally, the observed activation of ribosome recycling factor ( $f r r$ ) has been described during abortive initiation mechanism (peptidyl drop-off), leading to ribosome reuse ${ }^{33}$.

This trend points to a deep reorientation in protein synthesis rather than a general shutdown, and likely explains the dual expression pattern observed here, in which a part of the translation remains active while another seems to undergo a massive arrest. 

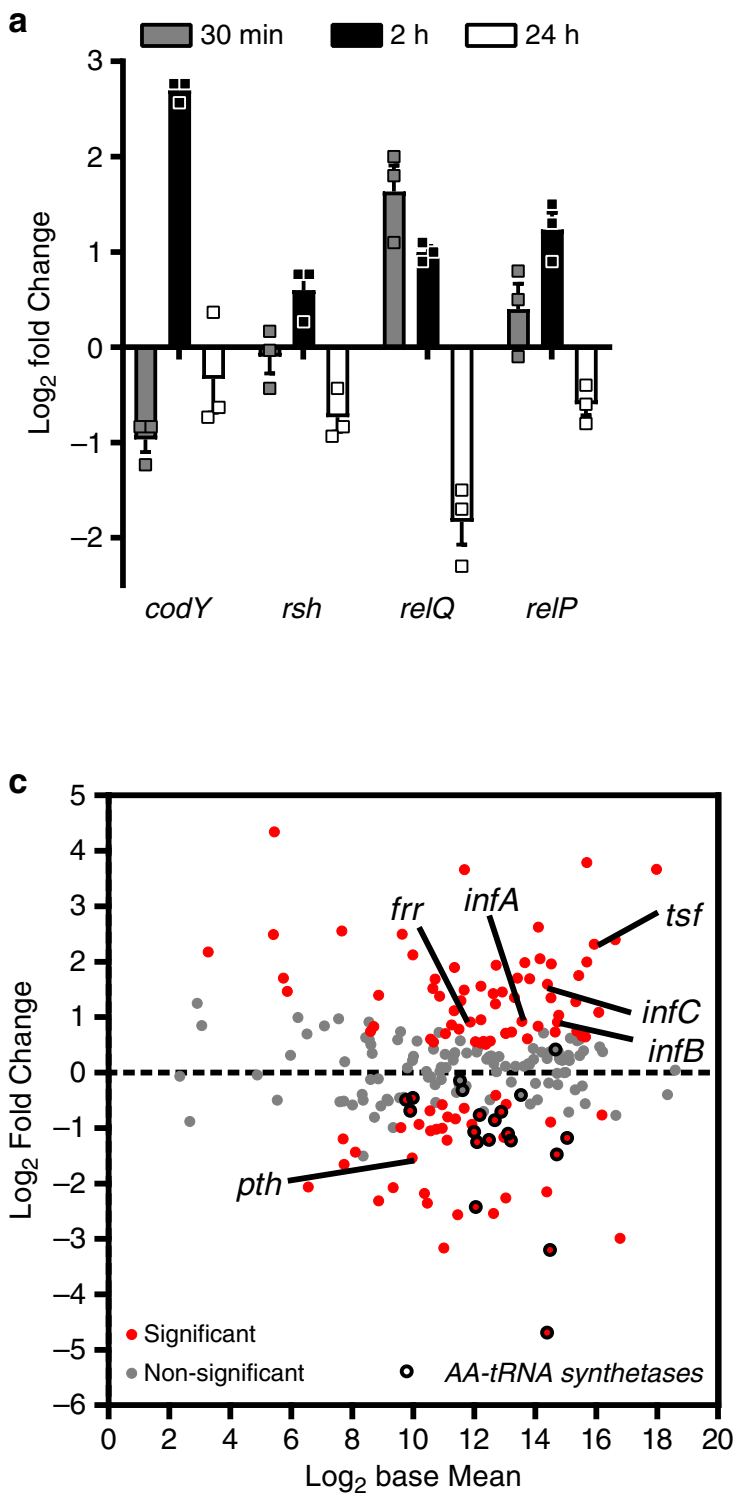
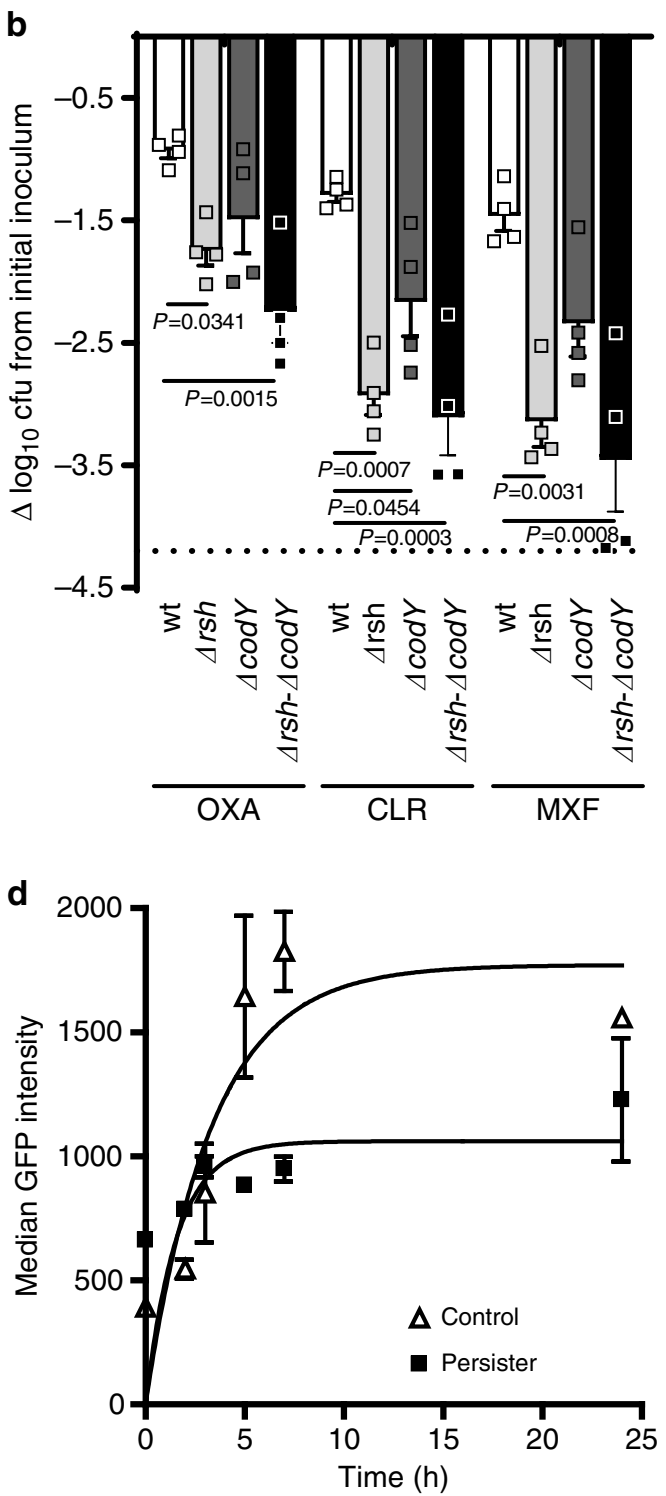

Fig. 3 A transitory boost of stringent response contributes to initiate the switch to intracellular persistence. a Quantitative real-time PCR of transcripts of stringent response regulators in intracellular persisters exposed to 50× MIC oxacillin for the indicated times. Data, expressed in fold change vs control samples (extracellular bacteria mixed with $\mathrm{J} 774$ cells lysate), are means \pm SEM of three independent experiments. b Cfus recovered from macrophages infected by HG001 (WT) and its isogenic mutants and exposed to $50 \times$ MIC oxacillin, clarithromycin or moxifloxacin for $24 \mathrm{~h}$. Data (expressed as reduction from the original inoculum) are means \pm SEM of four independent experiments. The dotted line indicates the limit of detection. Statistical significance was determined by one-way ANOVA with Dunnett's post-test. Oxacillin [OXA], clarithromycin [CLR], moxifloxacin [MXF]. c MA-plot of genes related to translation ${ }^{78}$. The graph displays the $\log _{2}$ Fold Change expression as a function of $\log _{2}$ Base Mean (mean expression signal across all samples). Typical members of the function are pointed and aminoacyl-tRNA synthetases are shown in black. The dotted line indicates the basal expression level in control samples. Statistical significance is based on adjusted $P$-value. $\mathbf{d}$ Rate of GFP synthesis in intracellular $S$. aureus. Macrophages were infected by non-induced bacteria for $24 \mathrm{~h}$, with (persisters) or without (control) 50× MIC oxacillin, and then induced for GFP expression for the indicated periods. Data are means \pm SEM of GFP signal from flow cytometric profiles from three independent experiments. $\mathbf{a}, \mathbf{b}, \mathbf{d}$, Source data are provided as a Source Data file.

To check for the functionality of protein synthesis, we measured the neo-synthesis of GFP as an indication of the translation rate. Intracellular persisters selected by oxacillin responded to induction by producing GFP, indicating that persisters display reduced, but active translation (Fig. 3d). These results led us to conclude that persisters are still metabolically active and that inhibition of translation is not sufficient to explain the antibiotic tolerance of intracellular $S$. aureus.

Persistence is not triggered by ATP or amino acid limitation. Persistence has been extensively studied in nutrient-poor models, mostly amino acid deprivation, in which bacteria tend to inhibit protein synthesis and promote amino acid biosynthesis. In stationary planktonic cultures, where bacteria are usually observed as dormant, ATP limitation has also been proposed to induce persistence $^{34}$. Recently, stimulation of production of reactive oxygen species by macrophages has been shown to reduce ATP levels and to increase antibiotic tolerance ${ }^{35}$.

We therefore measured ATP content in intracellular persisters released from macrophages, and found no significant difference from control samples (Fig. 4a), in line with a study showing that ATP content is not decisive for persister formation in $S$. aureus stationary cultures ${ }^{36}$. These data suggest that intracellular 
a

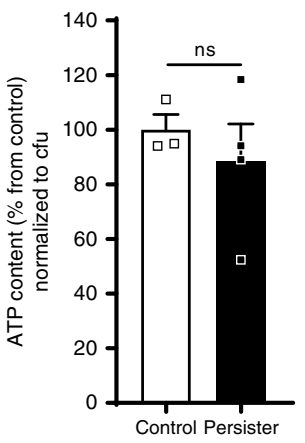

C
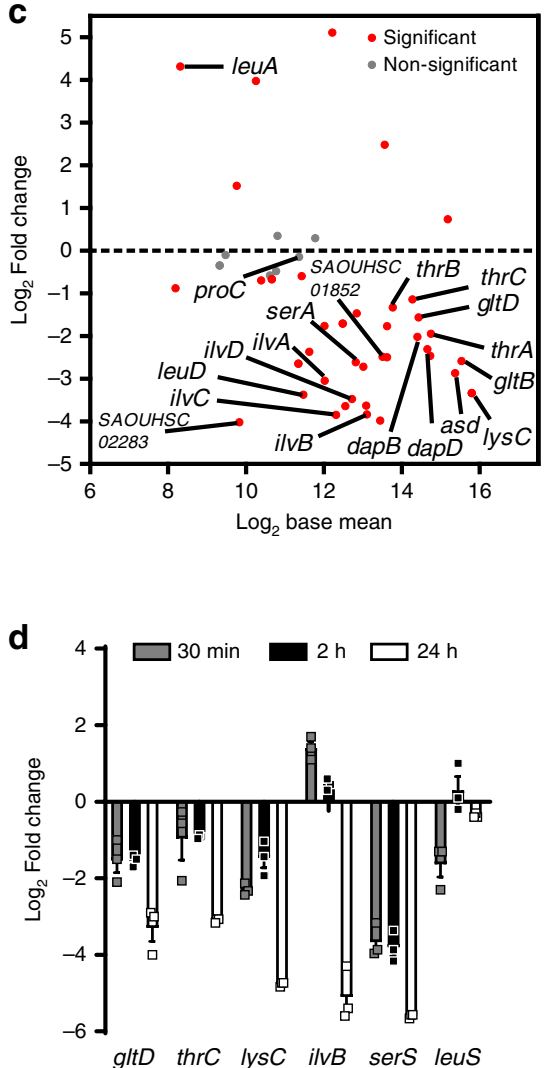

b

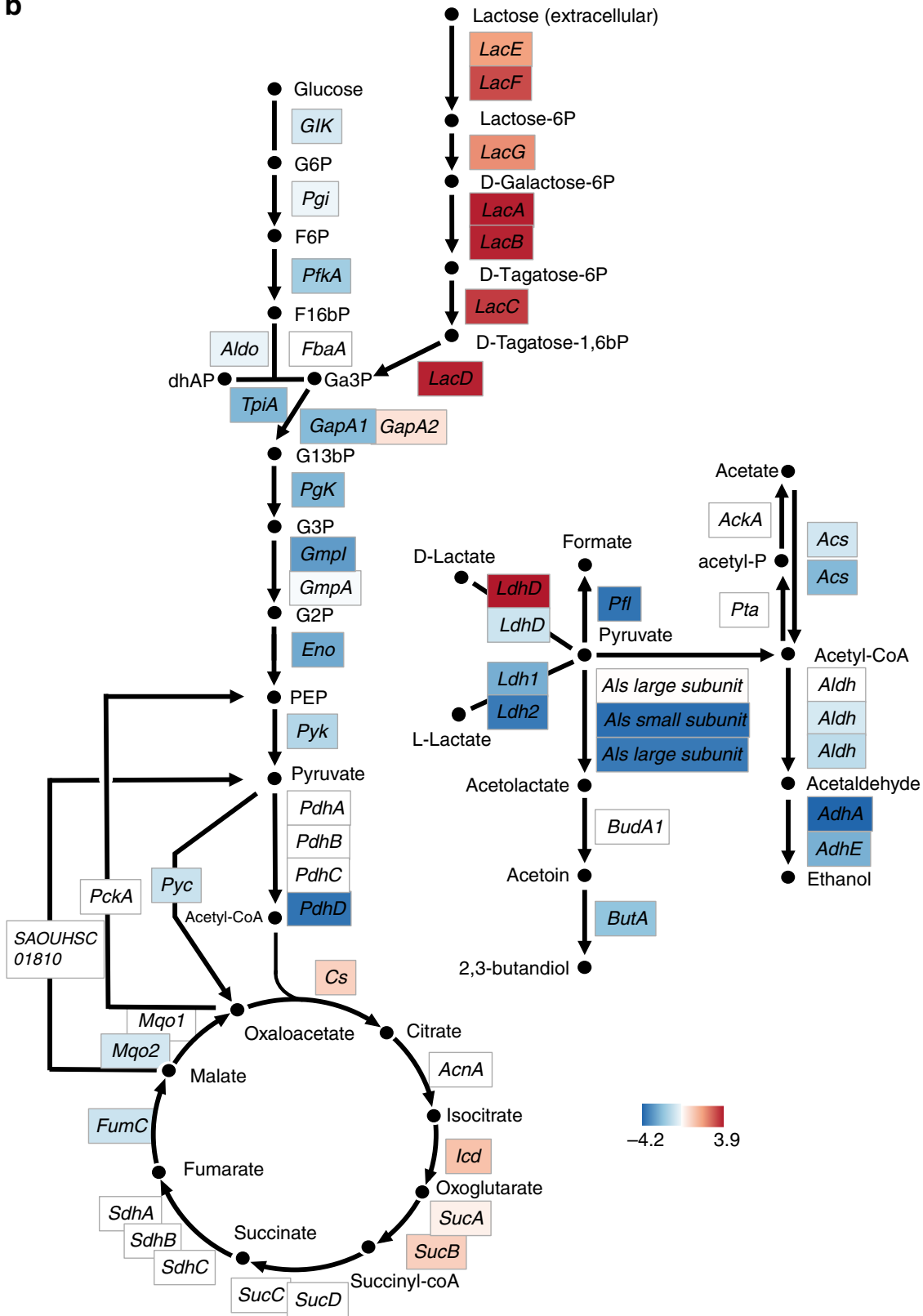

Fig. 4 Intracellular persister formation is not triggered by amino acid limitation nor ATP depletion. a Intrabacterial ATP concentration in intracellular persisters (exposed to 50× MIC oxacillin for $24 \mathrm{~h}$ ) and control samples (extracellular bacteria mixed with J774 cells lysate). Appropriate controls were performed to ensure the absence of contamination by eukaryotic ATP. Data are means \pm SEM of three independent experiments. Statistical significance was determined by two-tailed Student's $t$-test. ns non-statistically significant. b Schematic pathway of genes related to central carbon metabolism, annotated according to KEGG orthology ${ }^{78}$ and Genbank database ${ }^{23}$ and their $\log _{2}$ Fold Change expression levels. c MA-plot of genes related to amino acid metabolism within the stringent response stimulon (Supplementary Fig. 4; see reference ${ }^{55}$ ). The graph displays the $\log _{2}$ Fold Change expression as a function of $\log _{2}$ Base Mean (mean expression signal across all samples). Genes prominently activated after amino acid depletion 24 are pointed. The dotted line indicates the basal expression level in control samples. Statistical significance is based on adjusted $P$-value. $\mathbf{d}$ Quantitative real-time PCR of transcripts of genes related to amino acid synthesis in intracellular persisters exposed to 50x MIC oxacillin for the indicated periods. Data are means \pm SEM of three independent experiments. $\mathbf{a}$, d Source data are provided as a Source Data file.

persisters are metabolically active and have adapted their metabolism for ATP maintenance.

The expression pattern of the central metabolic flux (Fig. 4b) reveals metabolic network alterations: we found altered transcripts levels for glycolysis-related enzymes and evidence of an important carbon source shift between glucose and lactose, described as a trigger factor for persistence ${ }^{37}$. This result is in agreement with those of Traxler et al. ${ }^{38}$ who showed that E. coli persisters could be formed through a glucose-lactose diauxie in nutrient-rich conditions. Intracellular persisters also drastically adapt their respiration status, with an expression program resembling that observed in anaerobiosis. As revealed by enrichment analysis, genes related to oxidative phosphorylation (Supplementary Fig. 6) were mostly repressed ${ }^{39}$, to the benefit of massive D-lactate fermentation. As the expression levels of TCA cycle genes were rather maintained under these conditions, they could contribute to the redox balance for sustained fermentation. 
The transcription signature of the genes related to amino acid metabolism within the SR stimulon (Fig. 4c) revealed that the vast majority were silent after $24 \mathrm{~h}$, and also at earlier infection stages (Fig. 4d), when the transcription of SR regulators is already taking place. We conclude that amino acid limitation is not a trigger factor for intracellular persistence. Because these persisters sustain protein synthesis, we may suppose bacteria rely on other resource pools available in host cell, thereby ruling out the vacuolar nutrient-poor model ${ }^{40,41}$.

Although coherent with studies in Gram-negative pathogens ${ }^{42}$, these conclusions are still under debate for S. aureus, for which only amino acid limitation has been proposed as a trigger factor for intracellular persistence ${ }^{24}$.

Redundant adaptive responses lead to multidrug tolerance. It was previously hypothesized that a central growth arrest would lead to the inactivation of antibiotic targets and to tolerance. Such corrupted targets would prevent fluoroquinolones from generating DNA breaks, aminoglycosides from causing protein mistranslation, or $\beta$-lactams from impairing peptidoglycan reticulation ${ }^{42}$. Yet, our model denies pure dormancy of persisters but rather argues for a switch, partly initiated by SR, to a state where active processes ensure functional bacterial maintenance.

To better understand factors that underlie tolerance, we first examined how persisters induced by one drug would reply to another one. To this effect, intracellular persisters induced by oxacillin for $24 \mathrm{~h}$ were then exposed to fluoroquinolones, macrolides or aminoglycosides for an additional $24 \mathrm{~h}$ period in the continuing presence of oxacillin (Fig. 5a). When combined with oxacillin, all three antibiotics led to higher reductions in persister counts than oxacillin during the entire duration of the experiment and the kinetics of killing remained biphasic (Supplementary Fig. 7). Strikingly, no additional decrease was observed when the second drug was added after exposure to oxacillin. This indicates that oxacillin was able to induce a phenotype conferring a general tolerance to the four antibiotic classes, irrespective of their mechanism of action.

This observation strongly suggests that persister and tolerant phenotype occurs through multiple protective mechanisms intracellularly.

The gene expression of peptidoglycan biosynthesis is significantly reprogrammed within intracellular persisters (Fig. 5b), matching with an activation of the cell wall stress stimulon (CWSS), a protective response to cell wall defects and cell wallactive antibiotics ${ }^{43}$. These changes include the induction of genes within the core of the CWSS: the two-component system vraS/ $v r a R$, and genes involved in the late steps of peptidoglycan synthesis (i.e., $p b p 2$, the transglycosylase $s g t B$, and $f m t A$ encoding a penicillin binding protein with low affinity to $\beta$-lactams). CWSS induction provides a certain level of tolerance to most VraS/Rinducing agents ${ }^{43}$. Thus, intracellular persisters exhibit active responses for cell wall maintenance that likely mediate the observed tolerance to oxacillin.

Intracellular persisters also elicit an extensive program for preserving genome integrity through the SOS response. This network is a highly conserved DNA damage repair system which has been shown to induce tolerance to fluoroquinolones ${ }^{44}$ and confer protection against the bactericidal effects of $\beta$-lactams in $E$. $\operatorname{coli}^{45}$. In this pathway, RecA both facilitates recombinational repair and stimulates auto-cleavage of the repressor LexA, resulting in de-repression of genes involved in DNA repair or recombination ${ }^{46}$.

Within intracellular persisters, typical effectors of the SOS network, such as the excision repair systems or mismatch and repair systems, were largely transcribed (Fig. 5c, d) together with genes encoding fluoroquinolone targets, which may cooperatively contribute to the observed fluoroquinolone tolerance.

$S$. aureus persisters also undergo a massive transcription of the heat shock stimulon (Fig. 5e), a central response in stress tolerance. Under our conditions, dnaK, groESL, and grpE transcripts belong to the $98^{\text {th }}$ percentile of the fold change distribution in the transcriptome, thus indicating a drastic activation of this response. The DnaK and GroESL chaperone/ chaperonin sequentially function as a crucial bacterial protein folding machinery and participate in the degradation of defective proteins ${ }^{47}$. In the context of multiple stresses and dysregulated translation, its implication in bacterial tolerance is wide. By dealing with damaged proteins, this system is known to participate in tolerance to both $\beta$-lactams ${ }^{48}$ and aminoglycosides $^{49}$ and possibly influences the action of macrolides ${ }^{50}$. Macrolides target protein synthesis and cause early peptidyltRNA drop-off ${ }^{51}$. Although the mechanisms of macrolide tolerance are largely unknown, a role of this stimulon is conceivable since GroESL has been shown to positively affect the peptidyl-tRNA processing by peptidyl-tRNA hydrolase (Pth) ${ }^{33}$. Additionally, because the uptake of aminoglycosides requires proton motive force ${ }^{52}$, the deeply impaired transcription of the electron transport chain may favorably contribute to the tolerance to aminoglycosides, together with the decrease in translation rate, which has been proposed as a factor leading to tolerance towards protein synthesis inhibitors ${ }^{53}$ (Supplementary Table 2 for summary). Interestingly, this set of adaptive responses redundantly occurs in all permissive host cells tested (Supplementary Fig. 8).

To help clarify how this network of signaling pathways is taking place, and especially their relationship with SR, we studied the effect of rsh-codY mutation (HG001 strain and its isogenic mutant) on key determinants of CWSS, SOS and heat shock responses after $2 \mathrm{~h}$ of infection, i.e., a time point where SR was active (Fig. 5f). We found that SR positively modulated the expression pattern of SOS response, and, to a lesser extent, of CWSS through VraS. Alternatively, the expression of relP/relQ synthetases has been shown to be impacted by the VraS/R system $^{30}$. These mutations, nevertheless, did not annihilate the expression of these genes, and had no effect on heat shock chaperones, leading us to conclude that the SOS response and CWSS are positively modulated but not dependent on SR.

These findings are consistent with overlaps observed in transcription signatures between SR and CWSS or SOS stimulons. Indeed, relP has also been described as a core member of CWSS ${ }^{54}$ and conversely, $f m t A$ and $s g t B$, as members of SR stimulon, corroborating the entanglements in these responses. Similarly, lexA regulator belongs to SR stimulon ${ }^{55}$.

Additionally, in the absence of antibiotic pressure, intracellular bacteria displayed similar inductions of most of the determinants of SOS and heat shock responses, and a basal induction of CWSS, which becomes markedly induced upon antibiotic exposure (Supplementary Fig. 9), suggesting that stresses imposed by the intracellular environment also contribute to the changes observed in these signaling networks.

\section{Discussion}

This work clearly demonstrates the presence of intracellular persisters of $S$. aureus during antibiotic exposure, especially by monitoring bacterial division at a single cell level. We show that antibiotic pressure allows for the induction of homogenous living and non-dividing populations of persisters and that this phenotype is stable but highly reversible upon drug removal. Our model thus indicates a very dynamic bidirectional switch to intracellular persistence. The intracellular environment, by allowing both 
a
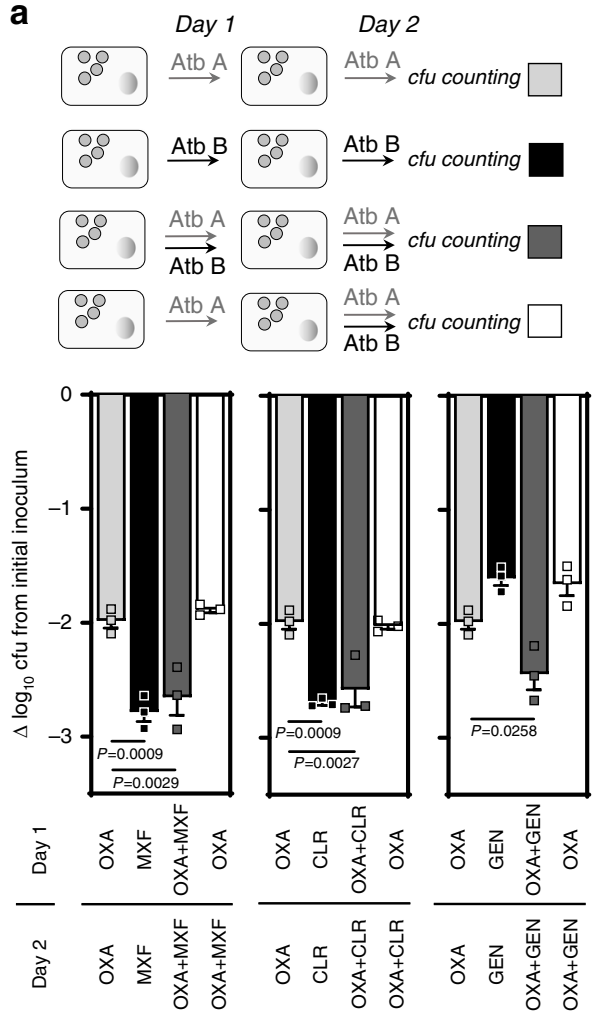

b

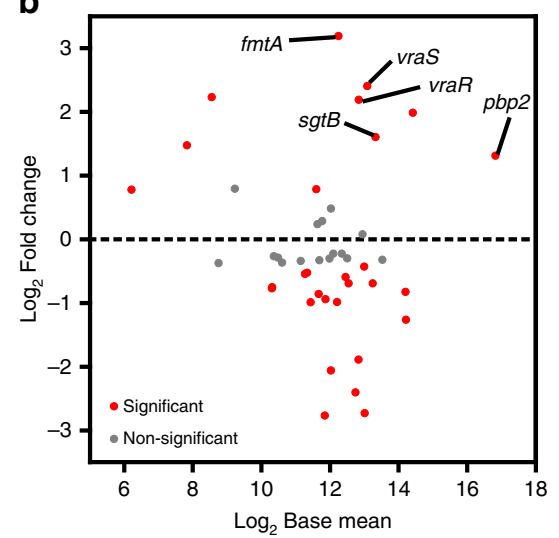

$\log _{2}$ Base mean

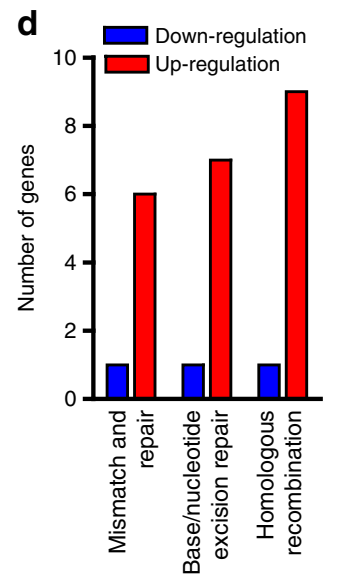

C

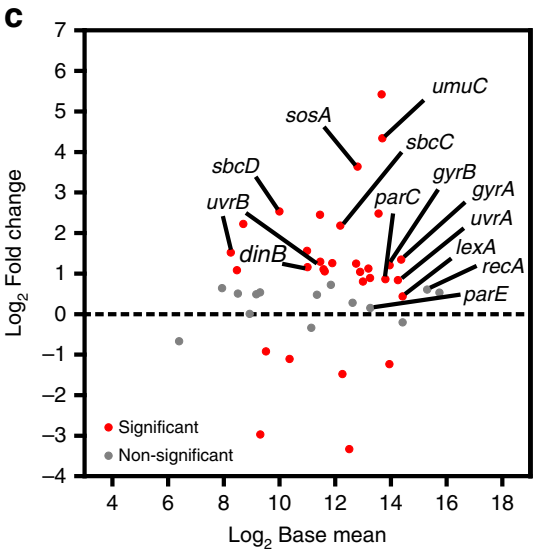

e

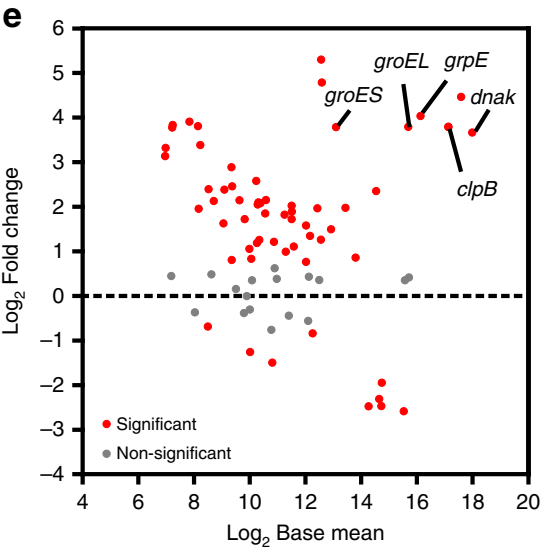

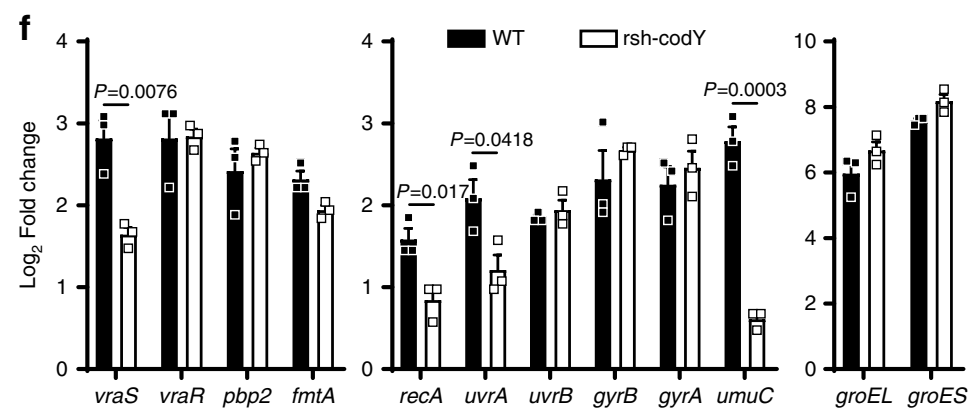

Fig. 5 A mosaic of redundant adaptive responses leads to multidrug tolerance. a Activity of antibiotic combinations added simultaneously or in succession against intracellular persisters. Intracellular persisters were challenged to 50× MIC of antibiotics alone or in combination, and recovered from macrophages and proceeded for cfu counting after $48 \mathrm{~h}$, following the experimental procedure described above. For combinations, antibiotics were added either at the same time as oxacillin or $24 \mathrm{~h}$ after oxacillin. Data (expressed as cfu reduction from the original inoculum) are means \pm SEM of three independent experiments. Statistical significance was determined by one-way ANOVA with Dunnett's post-test. Oxacillin [OXA], moxifloxacin [MXF], clarithromycin [CLR], gentamicin [GEN]. b, c MA-plots of genes related to peptidoglycan biosynthesis ${ }^{78}$ (extended to vraS/R and cell-envelope biogenesis genes from the cell wall stress stimulon) ${ }^{80}$ and SOS response stimulon 46,55 , respectively. The graphs display the $\log _{2}$ Fold Change expression as a function of $\log _{2}$ Base Mean (mean expression signal across all samples). Typical members of the stimulons are pointed. The dotted lines indicate the basal expression level in control samples. Statistical significance is based on adjusted $P$-value. $\mathbf{d}$ Number of up- or downregulated DEGs related to DNA repair ${ }^{78}$. e MA-plot of genes related to heat shock stimulon ${ }^{55}$. $\mathbf{f}$ Quantitative real-time PCR of transcripts of determinants of CWSS, SOS response, and heat shock stimulon, from left to right, in HG001 (WT) or HG001 rsh-codY double mutants ( $\Delta$ rsh- $\Delta$ codY) exposed to $50 \times$ MIC oxacillin for $2 \mathrm{~h}$ of infection. Data are means \pm SEM of three independent experiments. Statistical significance was determined by two-tailed Student's $t$-test. a, $\mathbf{f}$ Source data are provided as a Source Data file.

persistence and replication, could then represent both a privileged reservoir for the pathogen and a major source of relapses after antibiotic removal.

Our RNA-sequencing analysis offers a comprehensive overview of the transcriptomic profile of the living population of S. aureus persisters. The transcriptomic patterns highlighted in this study led us to propose a model of factors leading to persistence and tolerance (Fig. 6).

A transitory boost of SR contributes to initiate the switch to intracellular persistence in response to a complex set of environmental stresses. Interestingly, no ATP or amino acid limitations occur intracellularly. Noteworthy, neither oxacillin in 


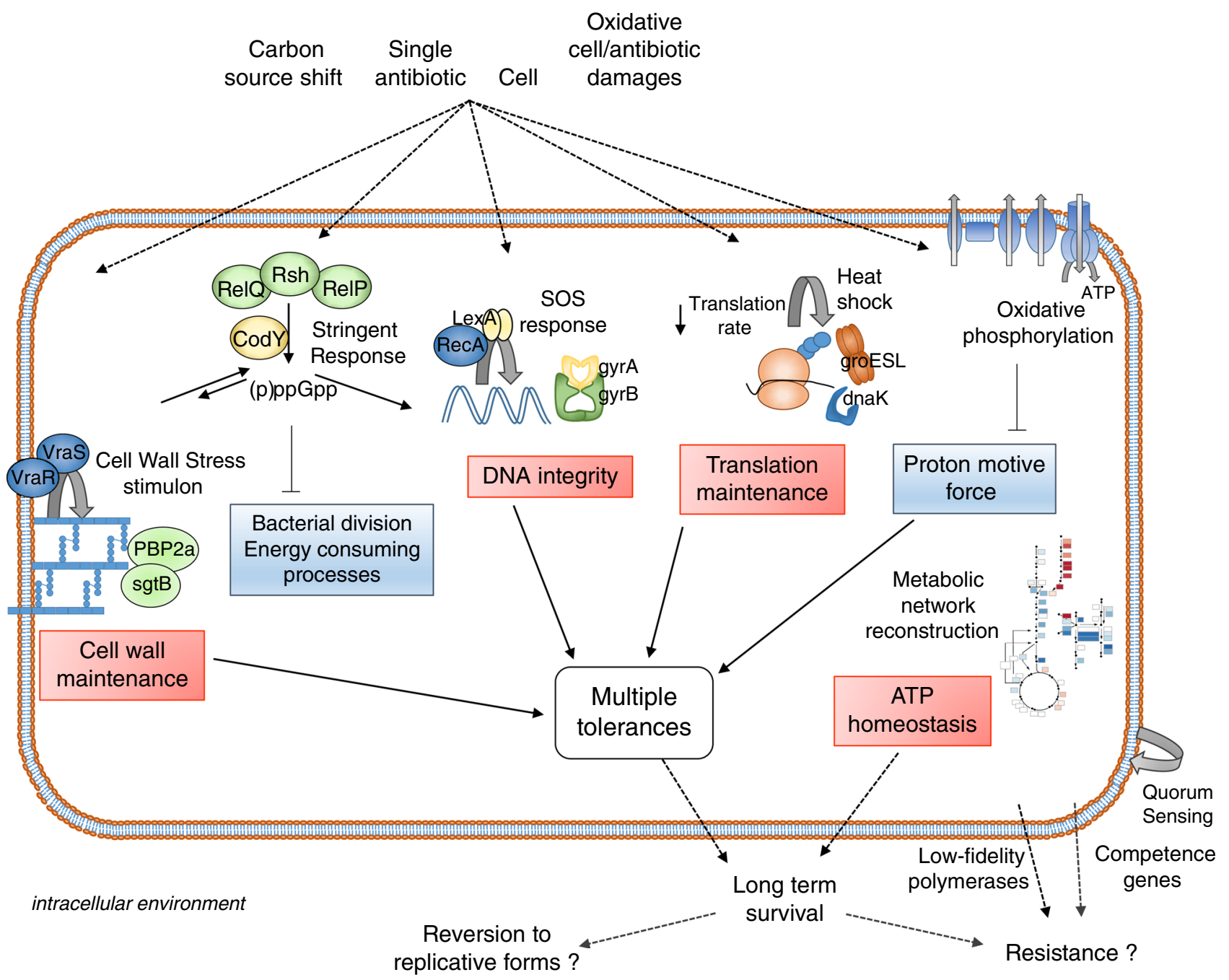

Fig. 6 Overview of intracellular persistence regulation of S. aureus. In vacuolar nutrient-rich compartments, persisters are metabolically active cells shielding cell wall, DNA and translation products. Under pressure of the environmental factors from the host cell, including a carbon source shift and antibiotic pressure, persisters promote a network of stress or adaptive responses displaying multiple entries. Stringent response does not show signs of activity for prolonged periods but rather contributes partly to initiate the switch to a persister phenotype through (i) post-translational modifications, contributing to an almost immediate blockade of bacterial division, and (ii) transcriptional regulation, silencing energy-consuming processes. Regulation circuits also include the cell wall stress stimulon, the SOS response, and the heat shock response. These active responses, together with a decrease in oxidative phosphorylation and in translation levels, lead to multidrug tolerance upon exposure to a single antibiotic. This stable phenotype allows bacteria to maximize the chances of long-term survival. Finally, depending on the level of stress, this state could either revert to replicative forms, or promote the evolution to resistant forms, through increased probability of mutations and horizontal gene transfer.

planktonic cultures nor permissive cells alone could trigger marked $S$. aureus persistence under our conditions, thus indicating that stresses of different nature can collectively initiate persistence when reaching a certain threshold. During infection, bacteria are typically internalized in vacuolar compartments and experience numerous stresses from the host cell and antibiotic pressure. Besides the direct effect of the antibiotic, these stresses include a carbon source transition and a contribution of acidic $\mathrm{pH}^{14}$, which may work in concert with cell- and antibioticinduced oxidative stresses $^{35,56}$.

SR redirects many physiological activities at the expense of those required for growth and proliferation through transcriptional regulation circuits and post-translational modifications that represent a second control point "freezing" the system, conferring extreme plasticity towards external stresses.

We did not find evidence of expression of SR regulators for prolonged periods. Our data rather supports the hypothesis that the mechanism of persistence is not solely dependent on SR but that multiple pathways contribute to persister generation and maintenance. The redundant character of this signaling network might thus result from the continuous and multiple stresses bacteria are facing, rather than a unique regulator.

Our work also questions the concept of tolerance occurring as a passive phenomenon through target inactivity. We showed that intracellular persisters are metabolically active cells, which exhibit a mosaic of adaptive responses that lead to a phenotype of multiple tolerance. These responses seem to redundantly occur, and involve the activation of the CWSS, the SOS and heat shock responses, as well as the induction of several antibiotic targets. This study also demonstrates that exposure to a single drug can trigger a phenotype of multiple tolerance to several antibiotic classes intracellularly. The clinical meaning of these observations remains to be established.

Our model for persistence integrates apparent contradictory observations (e.g., dormancy status or levels of tolerance) often interpreted as physiological diversity 57,58 (Supplementary Fig. 10). 
We propose that persistence is highly plastic through essentially redundant regulations, which differentially adapt levels of dormancy as a function of sensed level of stress. In that sense, the proposed heterogeneity should essentially result from differences of degree rather than of nature.

Once intracellular persistence is established, we hypothesize this could constitute a state at the crossroads, either reverting to replicative forms if the level of stress is released, or promoting the evolution to resistant forms if the pressure is maintained ${ }^{59}$. In the latter case, our data indicate that this evolution could be promoted mainly at two distinct levels, through potentiation of higher mutation rates ${ }^{60}$ and extensive horizontal gene transfer ${ }^{61}$, as a consequence of drastic activations of low-fidelity polymerases and competence genes, respectively (Supplementary Fig. 11 and Supplementary Table 3 for summary).

Thus, the present work demonstrates that bacteria surviving to antibiotics intracellularly are persisters which harbor a profoundly reshaped transcriptome. They activate a series of stress responses for long-term survival. Our results therefore highlight persistence as a potential critical trigger for therapeutic failures.

\section{Methods}

Bacterial strains and cells. Strains used in this study are listed in Supplementary Table 1. S. aureus strains were routinely grown at $37^{\circ} \mathrm{C}$ in cation-adjusted MuellerHinton broth (MHB-CA; Sigma) under shaking at $300 \mathrm{rpm}$. The fully susceptible strain SH1000 (ref. ${ }^{62}$ ) was used to harbor the pALC2084 plasmid, which encodes a reporter $g f p$ gene cloned downstream of a $x y l / t e t O$ promoter and allows a dosedependent tetracycline induction in vivo and in vitro ${ }^{63}$. GFP production was induced by a sub-inhibitory concentration $\left(125 \mathrm{ng} \mathrm{mL}^{-1}\right)$ of tetracycline. Overnight cultures were supplemented with $10 \mathrm{mg} \mathrm{L}^{-1}$ chloramphenicol. All experiments were performed on SH1000, unless stated otherwise.

Murine J774A.1 macrophages ${ }^{64}$ (Sandoz Forschung Laboratories) were cultured in RPMI 1640 medium (Thermo Fisher Scientific) supplemented with 10\% fetal bovine serum (FBS; Thermo Fisher Scientific) at $37^{\circ} \mathrm{C}$ in a $5 \% \mathrm{CO}_{2}$ atmosphere When indicated, cells were washed in sterile PBS (filtered on $0.22 \mu \mathrm{m}$ pore size membrane when used for flow cytometry analysis). Cells were seeded in 12-well plates (Greiner bio-one), in Labtek 2-well Chamber Slide (Nunc) for confocal microscopy, or in $145 \mathrm{~mm}$ cell culture dishes (Greiner bio-one) for cell sorting.

Human macrophages were obtained by isolation and differentiation of monocytes from peripheral blood according to the protocol from Menck et al. ${ }^{65}$. Briefly, buffy coats from healthy blood donors were subjected to a double Ficoll and Percoll density gradient centrifugation for isolation of monocytes from peripheral blood. For differentiation to macrophages, monocytes were resuspended in RPMI 1640 with $2 \%$ human serum (Biowest), $1 \%$ penicillin/streptomycin (Thermo Fisher Scientific) and $2.5 \mathrm{ng} \mathrm{mL}^{-1} \mathrm{M}$-CSF (Miltenyl Biotec) and seeded in 12-well plates for 7 days at $37^{\circ} \mathrm{C}$ in a $5 \% \mathrm{CO}_{2}$ atmosphere. Cells were then collected and seeded in 12 -well plates in RPMI 1640 with $10 \%$ FBS for infection. Human THP-1 monocytes (ATCC TIB-202) were cultured in RPMI 1640 medium with 10\% FBS as previously described ${ }^{66}$. Human MG63 osteoblastic cells (LGC Standards) were cultured in Dulbecco's modified Eagle's medium (DMEM) with $10 \% \mathrm{FBS}^{67}$. Human A549 (ATCC CCL-185) and MCF7 (ATCC HTB-22) epithelial cells were cultured in DMEM with $10 \%$ FBS and DMEM with $10 \%$ FBS and $0.01 \mathrm{mg} \mathrm{mL}^{-1}$ insulin (Gibco). Human adult primary keratinocytes were cultured in supplemented EpiLife medium (EpiLife with S7; Thermo Fisher Scientific) in collagen coated plates as previously described ${ }^{68}$.

Experiments in broth. For Fluorescence dilution experiments, overnight $S$. aureus cultures in MHB-CA supplemented with $125 \mathrm{ng} \mathrm{mL}^{-1}$ tetracycline were centrifuged at $5000 \mathrm{~g}$ for $5 \mathrm{~min}$, washed out in PBS to remove tetracycline, and diluted in fresh medium to reach a starting $\mathrm{OD}_{620 \mathrm{~nm}}$ of 0.05 . Cultures were incubated at $37^{\circ} \mathrm{C}$ and aliquots were collected over time, centrifuged, washed in PBS, resuspended in filtered PBS and analyzed by flow cytometry or epifluorescence microscopy (Carl Zeiss Axioskop 40) as described below (see also Supplementary Fig. 12 for gating methods). For time-kill curves, S. aureus was grown overnight in MHB-CA, diluted in fresh medium to reach a starting $\mathrm{OD}_{620 \mathrm{~nm}}$ of 0.05 and grown to the mid-exponential phase. Cultures were diluted to a starting inoculum of $1 \times$ $10^{6} \mathrm{cfu} \mathrm{mL}^{-1}$ and exposed to oxacillin (Sigma), clarithromycin (SMB-Galephar), or moxifloxacin (Sigma) at 50× their respective MIC, for the indicated times. For cfu counting, samples were diluted in PBS before plating on Tryptic Soy agar. Data are expressed as $\log _{10} \mathrm{cfu} \mathrm{mL}^{-1}$ after the incubation period compared to the starting inoculum.

Infection of macrophages and other cell types. Infection was performed following a protocol adapted from Seral et al. ${ }^{69}$ and Barcia-Macay et al. ${ }^{66}$. S. aureus was grown overnight in MHB-CA supplemented with $125 \mathrm{ng} \mathrm{mL}^{-1}$ tetracycline.
Bacteria were then centrifuged at $5000 \mathrm{~g}$ for $5 \mathrm{~min}$ and resuspended in RPMI 1640 supplemented with $125 \mathrm{ng} \mathrm{mL}^{-1}$ tetracycline and $10 \%$ human serum to allow opsonization for $30 \mathrm{~min}$ at $37^{\circ} \mathrm{C}$. Bacteria were centrifuged, resuspended in fresh RPMI 1640 with $10 \%$ FBS and $125 \mathrm{ng} \mathrm{mL}^{-1}$ tetracycline, and incubated with cells during $30 \mathrm{~min}$ at $37^{\circ} \mathrm{C}$ at a multiplicity of infection of $10: 1$ to allow phagocytosis The multiplicity of infection (MOI) was adapted to 100:1 for RNA-seq in order to obtain a sufficient amount of bacterial material (see Supplementary Fig. 13 for evaluation of the absence of effect of MOI on the dynamics of replication) and 1:1 for confocal microscopy. Cells were then washed with PBS, and non-phagocytized bacteria were eliminated by a $40 \mathrm{~min}$ incubation at $37^{\circ} \mathrm{C}$ in RPMI 1640 supplemented with $50 \mathrm{mg} \mathrm{L}^{-1}$ gentamicin (Sigma). Gentamicin was eliminated by washing in PBS, after which cells were reincubated at $37^{\circ} \mathrm{C}$ in RPMI 1640 with $10 \%$ FBS in the presence either of $2 \times$ MIC of gentamicin (to prevent extracellular growth; control conditions) ${ }^{69}$, or of oxacillin, clarithromycin, or moxifloxacin at 2 or $50 \times$ their respective MIC, for the indicated times. Cells were then washed with PBS, scrapped and lysed with PBS containing 0.1\% (w/v) Triton X-100 (Sigma) to release intracellular bacteria. Lysates were centrifuged at $300 \mathrm{~g}$ for $5 \mathrm{~min}$ to discard cellular debris. Bacteria were collected by centrifugation at $5000 \mathrm{~g}$ for $5 \mathrm{~min}$, washed in PBS, and resuspended in filtered PBS. For cfu counting, samples were diluted in PBS before plating on Tryptic Soy agar. Data are expressed as $\log _{10}$ cfu per mg cell protein after the incubation period compared to the post-phagocytosis inoculum. The same protocol was applied for infection of primary human macrophages and THP-1 monocytes, except that THP-1 cells were growing in suspension, so that washing procedures involved cell pelleting by centrifugation $(7 \mathrm{~min}, 300 \mathrm{~g}$ ). For other cell types (A549, MCF7, MG63 and primary keratinocytes), the same protocol was applied as for macrophages, except that bacteria were incubated with cells during $2 \mathrm{~h}$ at a multiplicity of infection of 50:1. This allowed to reach typical inocula of $2 \times 10^{6}$ bacteria $\mathrm{mg}^{-1}$ of cell protein for macrophages and monocytes, $0.4 \times 10^{6}$ bacteria per mg of cell protein for A549, MCF7 and MG63, and $0.2 \times 10^{6}$ bacteria per $\mathrm{mg}$ of cell protein for keratinocytes. All experiments were performed on SH1000 induced for GFP expression, with the exception of experiments on the rate of GFP synthesis (see hereafter).

Rate of GFP synthesis in intracellular persisters. Macrophages were infected by non-induced bacteria following the same procedure and incubated with or without $50 \times$ MIC oxacillin for $24 \mathrm{~h}$, after which induction were performed with $125 \mathrm{ng} \mathrm{mL}^{-1}$ tetracycline for the indicated periods.

Confocal microscopy. Infected macrophages seeded in Labtek chambers were incubated with gentamicin or moxifloxacin in RPMI 1640 without phenol red. Prior to microscopy, chambers were removed and slides covered with cover glasses. Infected cells were observed with a Cell Observer SD (Carl Zeiss) and analyzed with Zen v1.1.2.0 software (Carl Zeiss).

Flow cytometry. S. aureus isolated from broth cultures or from macrophages were resuspended in filtered PBS, stained with $10 \mu \mathrm{g} \mathrm{mL}^{-1}$ propidium iodide, and analyzed using a FACSVerse cytometer (BD Biosciences) for GFP signal intensities (FITC channel, medium flow rate). Forward-scatter width (FCS-W) versus forward-scatter area (FSC-A), and side-scatter width (SSC-W) versus side-scatter area (SSC-A) were used to gate out damaged or multiplet cells. Of those, propidium iodide-positive bacteria were gated out. Data were analyzed with FlowJo 10.5.2 software (TreeStar Inc.). The level of replication of the population ( $F$, fold replication) is calculated by the ratio $\mathrm{Me}_{0} / \mathrm{Me}_{\mathrm{t}}$ with Me being the median GFP intensity of the bacterial population at a given time. The number of generations, $N$, is deduced from $F=2^{N}$ (ref. ${ }^{19}$ ).

Samples preparation for RNA-seq. For differential expression analysis, bacterial reference samples (hereafter named "control samples" in RNA-seq experiments) were collected from an overnight culture (MHB-CA supplemented with $125 \mathrm{ng} \mathrm{mL}^{-1}$ tetracycline) by centrifugation at $5000 \mathrm{~g}$ for $5 \mathrm{~min}$, resuspended in RPMI 1640 and incubated for $30 \mathrm{~min}$ at $37^{\circ} \mathrm{C}$. This bacterial suspension was then mixed with a cell lysate obtained from non-infected macrophages incubated in RPMI 1640 with $10 \%$ FBS for $24 \mathrm{~h}$, in order to mimic the matrix effect of macrophages in the persister condition. The relative amount of bacteria and cells was adjusted to that obtained in $24 \mathrm{~h}$-infected cells. Intracellular persisters were collected from macrophages as described for flow cytometry. Both control and persisters conditions were proceeded for the same sorting procedure. Bacteria were immediately proceeded for sorting on the basis of their propidium iodide profile and GFP expression level using the gating methods described above, in a FACSAria III cytometer operated by the BD FACSDiva 8.0.1 software (BD Biosciences), under continuous cooling to $4{ }^{\circ} \mathrm{C}$ (including the input tube holder and the collection tube) at high flow rate. Samples were then immediately processed for RNA extraction.

RNA extraction. S. aureus recovered from infected macrophages or from control samples were centrifuged at $5000 \mathrm{~g}$ for $5 \mathrm{~min}$ and lysed using the following procedure: pellets were resuspended in Tris-EDTA buffer containing freshly prepared $13 \mathrm{mg} \mathrm{mL}^{-1}$ lysozyme (Sigma) and $130 \mu \mathrm{g} \mathrm{mL}^{-1}$ lysostaphin (Sigma) for $30 \mathrm{~min}$ at room temperature. Resulting suspensions were processed for total RNA extraction 
with RNA extraction InviTrap Spin Universal RNA Mini Kit (Stratec) following the manufacturer's instructions. Traces of contaminating genomic DNA were removed from samples by treatment with TURBO DNase (Ambion) for $30 \mathrm{~min}$ at $37^{\circ} \mathrm{C}$ according to the manufacturer's instructions. RNA purity was checked using a NanoDrop spectrophotometer (Thermo Fisher Scientific).

RNA sequencing. Total RNA from three independent replicates were checked on the Bioanalyser system (Agilent) for its quality and integrity. Ribosomal RNA depletion was performed using the Bacteria RiboZero kit (Illumina). From rRNAdepleted RNA, directional libraries were prepared using the TruSeq Stranded mRNA Sample preparation kit following the manufacturer's instructions (Illumina). Libraries were checked for quality on Bioanalyser DNA chips Bioanalyser (Agilent). Quantification was performed with the fluorescent-based quantitation Qubit dsDNA HS Assay Kit (Thermo Fisher Scientific). Sequencing was performed as an SRM run (SR: Single Read, PE: Paired-end Reads, M: multiplexed samples) for 65 bp sequences on HiSeq 2500 Illumina sequencer $(65$ cycles). The multiplexing level was 6 samples per lane. Bioinformatics analysis were performed using the RNA-seq pipeline from Sequana ${ }^{70}$. Reads were cleaned of adapter sequences and low-quality sequences using cutadapt version 1.11 (ref. ${ }^{71}$ ). Only sequences at least $25 \mathrm{nt}$ length were considered for further analysis. Bowtie version 0.12.7 (ref. ${ }^{22}$ ) with default parameters, was used for alignment on the reference genome (CP000253.1, NCBI). Genes were counted using featureCounts version 1.4.6-p3 (ref. ${ }^{73}$ ) from Subreads package (parameters: -t gene -g ID -s 1). Count data were analyzed using $\mathrm{R}$ version 3.4.1 (ref. ${ }^{74}$ ) and the Bioconductor package DESeq2 version 1.16 (ref. ${ }^{75}$ ). The normalization and dispersion estimation were performed with DESeq2 using the default parameters and statistical tests for differential expression were performed applying the independent filtering algorithm. A generalized linear model was set in order to test for the differential expression between the intracellular persisters and control conditions. Raw $P$ values were adjusted for multiple testing according to the Benjamini and Hochberg $(\mathrm{BH})$ procedure $^{76}$ and genes with an adjusted $p$ value lower than 0.05 were considered differentially expressed. For over-representation analysis, S. aureus KEGG gene-sets were downloaded thanks to the EnrichmentBrowser R package version 2.14.3 (organism code sao). All the 106 KEGG sets were then tested for the over-representation in differentially expressed genes using the Fisher statistical test. Only gene-sets with a FDR lower than 0.05 were considered significantly enriched.

Quantitative real-time PCR. Total bacterial RNA from infected macrophages or from control samples at different time points was isolated as described for RNAseq analyses. RNA was reverse transcribed using transcription first strand cDNA synthesis kit (Roche Applied Science) according to the manufacturer's instructions. Amplification reactions were performed with Sybr green IQ Supermix (Bio-Rad Laboratories) using an iCycler iQ single-color real-time PCR detection system (BioRad Laboratories). Fold changes in expression versus control condition were determined using the $2^{(-\Delta \Delta \mathrm{Ct})}$ method ${ }^{77}$ with $g m k$ as a housekeeping gene. Primers sequences are listed in Supplementary Table 4.

ATP measurements. Bacteria were released from macrophages as described above, washed in $50 \mathrm{mM}$ Tris-HCl, centrifuged at $5000 \mathrm{~g}$ for $5 \mathrm{~min}$ and processed for lysis following the same procedure as for RNA extraction. Control samples (as described for RNA-seq experiments) were used for comparison purposes. Bacterial lysates were incubated $2 \mathrm{~min}$ at $100^{\circ} \mathrm{C}$, centrifuged at $9600 \mathrm{~g}$ for $2 \mathrm{~min}$ and assayed for ATP measurements, using the ATP determination kit (Thermo Fisher Scientific) according to the manufacturer's instructions. Bioluminescence was measured using a SpectraMax M3 548 Microplate Reader (Molecular Devices).

Ethics statement. Experiments on blood material were performed in strict accordance with governmental and European legislation relative to blood, cell and tissues-related activities, and were approved by the ethical committee Comité d'Ethique Hospitalo-Facultaire Saint-Luc (CEHF Saint-Luc; permit no. B403201730810). Human blood was collected in Croix-Rouge de Belgique centers, from healthy volunteers who gave written informed consent, in accordance with procedures of Service Francophone du Sang de la Croix-Rouge de Belgique.

\section{Statistical analysis and curve fitting. For RNA-seq analyses, differential} expressions of transcripts were based on adjusted $P$-values with a threshold of statistical significance set to 0.05 . Genes descriptions were indicated as described in GenBank database ${ }^{23}$ and classified according to KEGG orthology ${ }^{78}$ and KEGG pathway $^{79}$ databases (organism code CP000253.1 and sao respectively). Curve fitting and statistical analyses were performed with GraphPad Prism versions 4.03 or 8.3.1, GraphPad InStat v3.10 (GraphPad Software), and JMP Pro version 13.1.0. Statistical differences were determined using unpaired two-tailed Student's $t$-tests or one-way ANOVA with Dunnett's post-tests for multiple comparisons. $P$-values strictly inferior to 0.05 were used to show statistical significance and are indicated in Figures. Non-statistically significant differences are indicated as "ns".

Reporting summary. Further information on research design is available in the Nature Research Reporting Summary linked to this article.

\section{Data availability}

The source data underlying Figs. 1a, b, e, h, i, 1, 3a, b, d, 4a, d, 5a and f are provided as a Source Data file. RNA-seq data reported in this study have been deposited at Gene Expression Omnibus under accession number GSE139659 [https://www.ncbi.nlm.nih. gov/geo/query/acc.cgi?acc=GSE139659]. All other relevant data are available from the corresponding author on request.

Received: 5 August 2019; Accepted: 2 April 2020;

Published online: 04 May 2020

\section{References}

1. Bigger, J. Treatment of staphylococcal infections with penicillin by intermittent sterilisation. Lancet 244, 497-500 (1944).

2. Brauner, A. et al. Distinguishing between resistance, tolerance and persistence to antibiotic treatment. Nat. Rev. Microbiol. 14, 320-330 (2016).

3. Balaban, N. Q. et al. Bacterial persistence as a phenotypic switch. Science 305, $1622-1625$ (2004).

4. Fauvart, M., De Groote, V. N. \& Michiels, J. Role of persister cells in chronic infections: clinical relevance and perspectives on anti-persister therapies. $J$. Med. Microbiol. 60, 699-709 (2011).

5. Svenningsen, M. S. et al. Birth and resuscitation of (p)ppGpp induced antibiotic tolerant persister cells. Sci. Rep. 9, 6056 (2019).

6. Harms, A. et al. Prophages and growth dynamics confound experimental results with antibiotic-tolerant persister cells. mBio 8, e01964-17 (2017).

7. Dawson, C. C., Intapa, C. \& Jabra-Rizk, M. A. "Persisters": survival at the cellular level. PLoS Pathog. 7, e1002121 (2011).

8. Fisher, R. A., Gollan, B. \& Helaine, S. Persistent bacterial infections and persister cells. Nat. Rev. Microbiol. 15, 453-464 (2017).

9. Wood, T. K., Knabel, S. J. \& Kwan, B. W. Bacterial persister cell formation and dormancy. Appl. Environ. Microbiol. 79, 7116-7121 (2013).

10. Mulcahy, L. R. et al. Emergence of Pseudomonas aeruginosa strains producing high levels of persister cells in patients with cystic fibrosis. J. Bacteriol. 192, 6191-6199 (2010).

11. Conlon, B. P. Staphylococcus aureus chronic and relapsing infections: evidence of a role for persister cells: an investigation of persister cells, their formation and their role in S. aureus disease. Bioessays 36, 991-996 (2014).

12. Dhar, N. \& McKinney, J. D. Mycobacterium tuberculosis persistence mutants identified by screening in isoniazid-treated mice. Proc. Natl Acad. Sci. USA 107, 12275-12280 (2010)

13. Goneau, L. W. et al. Selective target inactivation rather than global metabolic dormancy causes antibiotic tolerance in uropathogens. Antimicrob. Agents Chemother. 58, 2089-2097 (2014).

14. Helaine, S. et al. Internalization of Salmonella by macrophages induces formation of nonreplicating persisters. Science 343, 204-208 (2014).

15. Adams, K. N. et al. Drug tolerance in replicating mycobacteria mediated by a macrophage-induced efflux mechanism. Cell 145, 39-53 (2011).

16. Garzoni, C. \& Kelley, W. L. Return of the Trojan horse: intracellular phenotype switching and immune evasion by Staphylococcus aureus. EMBO Mol. Med. 3, 115-117 (2011).

17. Peyrusson, F., Tulkens, P. M. \& Van Bambeke, F. Cellular pharmacokinetics and intracellular activity of gepotidacin against Staphylococcus aureus isolates with different resistance phenotypes in models of cultured phagocytic cells. Antimicrob. Agents Chemother. 62, e02245-17 (2018).

18. Roostalu, J. et al. Cell division in Escherichia coli cultures monitored at single cell resolution. BMC Microbiol. 8, 68 (2008).

19. Helaine, S. et al. Dynamics of intracellular bacterial replication at the single cell level. Proc. Natl Acad. Sci. USA 107, 3746-3751 (2010).

20. Rollin, G. et al. Intracellular survival of Staphylococcus aureus in endothelial cells: a matter of growth or persistence. Front. Microbiol. 8, 1354 (2017).

21. Horn, J. et al. Inside job: Staphylococcus aureus host-pathogen interactions. Int. J. Med. Microbiol. 308, 607-624 (2018).

22. Jubrail, J. et al. Inability to sustain intraphagolysosomal killing of Staphylococcus aureus predisposes to bacterial persistence in macrophages. Cell Microbiol. 18, 80-96 (2016).

23. US National Library of Medicine. Staphylococcus aureus subsp. aureus NCTC 8325, complete genome. NCBI https://www.ncbi.nlm.nih.gov/nuccore/ CP000253.1 (2014).

24. Geiger, T. et al. The stringent response of Staphylococcus aureus and its impact on survival after phagocytosis through the induction of intracellular PSMs expression. PLoS Pathog. 8, e1003016 (2012).

25. Amato, S. M. \& Brynildsen, M. P. Persister heterogeneity arising from a single metabolic stress. Curr. Biol. 25, 2090-2098 (2015). 
26. Goormaghtigh, F. et al. Reassessing the role of type II toxin-antitoxin systems in formation of Escherichia coli type II persister cells. mBio 9, e00640-18 (2018).

27. Ramisetty, B. C. et al. What is the link between stringent response, endoribonuclease encoding type II toxin-antitoxin systems and persistence? Front. Microbiol. 7, 1882 (2016).

28. Sojka, L. et al. Rapid changes in gene expression: DNA determinants of promoter regulation by the concentration of the transcription initiating NTP in Bacillus subtilis. Nucleic Acids Res. 39, 4598-4611 (2011).

29. Hauryliuk, V. et al. Recent functional insights into the role of (p)ppGpp in bacterial physiology. Nat. Rev. Microbiol. 13, 298-309 (2015).

30. Geiger, T. et al. Two small (p)ppGpp synthases in Staphylococcus aureus mediate tolerance against cell envelope stress conditions. J. Bacteriol. 196, 894-902 (2014).

31. Geiger, T. et al. Role of the (p)ppGpp synthase RSH, a RelA/SpoT homolog, in stringent response and virulence of Staphylococcus aureus. Infect. Immun. 78, 1873-1883 (2010).

32. Reiss, S. et al. Global analysis of the Staphylococcus aureus response to mupirocin. Antimicrob. Agents Chemother. 56, 787-804 (2012).

33. Das, G. \& Varshney, U. Peptidyl-tRNA hydrolase and its critical role in protein biosynthesis. Microbiology 152(Pt 8), 2191-2195 (2006).

34. Conlon, B. P. et al. Persister formation in Staphylococcus aureus is associated with ATP depletion. Nat. Microbiol. 1, 16051 (2016).

35. Rowe, S. E. et al. Reactive oxygen species induce antibiotic tolerance during systemic Staphylococcus aureus infection. Nat. Microbiol. 5, 282-290 (2020).

36. Wang, Y. et al. Inactivation of TCA cycle enhances Staphylococcus aureus persister cell formation in stationary phase. Sci. Rep. 8, 10849 (2018).

37. Radzikowski, J. L. et al. Bacterial persistence is an active sigmaS stress response to metabolic flux limitation. Mol. Syst. Biol. 12, 882 (2016).

38. Traxler, M. F., Chang, D. E. \& Conway, T. Guanosine $3^{\prime}, 5^{\prime}$-bispyrophosphate coordinates global gene expression during glucose-lactose diauxie in Escherichia coli. Proc. Natl Acad. Sci. USA 103, 2374-2379 (2006).

39. Voyich, J. M. et al. Insights into mechanisms used by Staphylococcus aureus to avoid destruction by human neutrophils. J. Immunol. 175, 3907-3919 (2005).

40. Palazzolo-Ballance, A. M. et al. Neutrophil microbicides induce a pathogen survival response in community-associated methicillin-resistant Staphylococcus aureus. J. Immunol. 180, 500-509 (2008).

41. Halsey, C. R. et al. Amino acid catabolism in Staphylococcus aureus and the function of carbon catabolite repression. mBio 8, e01434-16 (2017).

42. Nguyen, D. et al. Active starvation responses mediate antibiotic tolerance in biofilms and nutrient-limited bacteria. Science 334, 982-986 (2011).

43. Dengler, V. et al. Induction kinetics of the Staphylococcus aureus cell wall stress stimulon in response to different cell wall active antibiotics. $B M C$ Microbiol. 11, 16 (2011).

44. Dorr, T., Lewis, K. \& Vulic, M. SOS response induces persistence to fluoroquinolones in Escherichia coli. PLoS Genet. 5, e1000760 (2009).

45. Miller, C. et al. SOS response induction by beta-lactams and bacterial defense against antibiotic lethality. Science 305, 1629-1631 (2004).

46. Cirz, R. T. et al. Complete and SOS-mediated response of Staphylococcus aureus to the antibiotic ciprofloxacin. J. Bacteriol. 189, 531-539 (2007).

47. Singh, V. K. et al. An insight into the significance of the DnaK heat shock system in Staphylococcus aureus. Int J. Med Microbiol. 302, 242-252 (2012).

48. Singh, V. K. et al. Role for $d n a K$ locus in tolerance of multiple stresses in Staphylococcus aureus. Microbiology 153, 3162-3173 (2007).

49. Cardoso, K. et al. DnaK and GroEL are induced in response to antibiotic and heat shock in Acinetobacter baumannii. J. Med. Microbiol. 59, 1061-1068 (2010).

50. Kannan, K. et al. The general mode of translation inhibition by macrolide antibiotics. Proc. Natl Acad. Sci. USA 111, 15958-15963 (2014).

51. Tenson, T., Lovmar, M. \& Ehrenberg, M. The mechanism of action of macrolides, lincosamides and streptogramin $\mathrm{B}$ reveals the nascent peptide exit path in the ribosome. J. Mol. Biol. 330, 1005-1014 (2003).

52. Kohanski, M. A., Dwyer, D. J. \& Collins, J. J. How antibiotics kill bacteria: from targets to networks. Nat. Rev. Microbiol. 8, 423-435 (2010).

53. Davis, B. D. Mechanism of bactericidal action of aminoglycosides. Microbiol. Rev. 51, 341-350 (1987).

54. Sobral, R. G. et al. Extensive and genome-wide changes in the transcription profile of Staphylococcus aureus induced by modulating the transcription of the cell wall synthesis gene murF. J. Bacteriol. 189, 2376-2391 (2007).

55. Anderson, K. L. et al. Characterization of the Staphylococcus aureus heat shock, cold shock, stringent, and SOS responses and their effects on log-phase mRNA turnover. J. Bacteriol. 188, 6739-6756 (2006).

56. Wang, X. \& Zhao, X. Contribution of oxidative damage to antimicrobial lethality. Antimicrob. Agents Chemother. 53, 1395-1402 (2009).

57. Orman, M. A. \& Brynildsen, M. P. Dormancy is not necessary or sufficient for bacterial persistence. Antimicrob. Agents Chemother. 57, 3230-3239 (2013).

58. Maisonneuve, E. \& Gerdes, K. Molecular mechanisms underlying bacterial persisters. Cell 157, 539-548 (2014).
59. Cohen, N. R., Lobritz, M. A. \& Collins, J. J. Microbial persistence and the road to drug resistance. Cell Host Microbe 13, 632-642 (2013).

60. Mesak, L. R., Miao, V. \& Davies, J. Effects of subinhibitory concentrations of antibiotics on SOS and DNA repair gene expression in Staphylococcus aureus. Antimicrob. Agents Chemother. 52, 3394-3397 (2008).

61. Fagerlund, A., Granum, P. E. \& Havarstein, L. S. Staphylococcus aureus competence genes: mapping of the SigH, ComK1 and ComK2 regulons by transcriptome sequencing. Mol. Microbiol. 94, 557-579 (2014).

62. Horsburgh, M. J. et al. sigmaB modulates virulence determinant expression and stress resistance: characterization of a functional rsbU strain derived from Staphylococcus aureus 8325-4. J. Bacteriol. 184, 5457-5467 (2002).

63. Bateman, B. T. et al. Evaluation of a tetracycline-inducible promoter in Staphylococcus aureus in vitro and in vivo and its application in demonstrating the role of sigB in microcolony formation. Infect. Immun. 69, 7851-7857 (2001)

64. Snyderman, R. et al. Biologic and biochemical activities of continuous macrophage cell lines P388D1 and J774.1. J. Immunol. 119, 2060-2066 (1977).

65. Menck, K. et al. Isolation of human monocytes by double gradient centrifugation and their differentiation to macrophages in teflon-coated cell culture bags. J. Vis. Exp. 91, e51554 https://doi.org/10.3791/51554 (2014).

66. Barcia-Macay, M. et al. Pharmacodynamic evaluation of the intracellular activities of antibiotics against Staphylococcus aureus in a model of THP-1 macrophages. Antimicrob. Agents Chemother. 50, 841-851 (2006).

67. Trouillet, S. et al. A novel flow cytometry-based assay for the quantification of Staphylococcus aureus adhesion to and invasion of eukaryotic cells. J. Microbiol. Methods 86, 145-149 (2011).

68. Lemaire, S. et al. Activity of moxifloxacin against intracellular communityacquired methicillin-resistant Staphylococcus aureus: comparison with clindamycin, linezolid and co-trimoxazole and attempt at defining an intracellular susceptibility breakpoint. J. Antimicrob. Chemother. 66, 596-607 (2011).

69. Seral, C., Van Bambeke, F. \& Tulkens, P. M. Quantitative analysis of gentamicin, azithromycin, telithromycin, ciprofloxacin, moxifloxacin, and oritavancin (LY333328) activities against intracellular Staphylococcus aureus in mouse J774 macrophages. Antimicrob. Agents Chemother. 47, 2283-2292 (2003).

70. Cokelaer, T., Desvillechabrol, D., Legendre, R. \& Cardon, M. 'Sequana': a set of snakemake NGS pipelines. J. Open Source Softw. 2, 352 https://doi.org/ 10.21105/joss.00352 (2017).

71. Martin, M. Cutadapt removes adapter sequences from high-throughput sequencing reads. EMBnet J. 17, 10-12 (2011).

72. Langmead, B. et al. Ultrafast and memory-efficient alignment of short DNA sequences to the human genome. Genome Biol. 10, R25 (2009).

73. Liao, Y., Smyth, G. K. \& Shi, W. featureCounts: an efficient general purpose program for assigning sequence reads to genomic features. Bioinformatics 30, 923-930 (2014).

74. R Core Team. R: a language and environment for statistical computing. $R$ Foundation for Statistical Computing https://www.R-project.org/ (2018).

75. Love, M. I., Huber, W. \& Anders, S. Moderated estimation of fold change and dispersion for RNA-seq data with DESeq2. Genome Biol. 15, 550 (2014).

76. Benjamini, Y. \& Hochberg, Y. Controlling the false discovery rate: a practical and powerful approach to multiple testing. J. R. Stat. Soc. Ser. B 57, 289-300 (1995).

77. Livak, K. J. \& Schmittgen, T. D. Analysis of relative gene expression data using real-time quantitative PCR and the 2(-Delta Delta C(T)) Method. Methods 25, 402-408 (2001).

78. KEGG Orthology. Staphylococcus aureus subsp. aureus NCTC8325. GenomeNet https://www.genome.jp/kegg-bin/get_htext?sao00001.keg (2018).

79. KEGG Pathway. Staphylococcus aureus subsp. aureus NCTC8325. GenomeNet https://www.genome.jp/kegg-bin/show_organism? menu_type=pathway_maps\&org=sao (2020).

80. Utaida, S. et al. Genome-wide transcriptional profiling of the response of Staphylococcus aureus to cell-wall-active antibiotics reveals a cell-wall-stress stimulon. Microbiology 149, 2719-2732 (2003).

\section{Acknowledgements}

The authors thank N. Dauguet (De Duve Institute, Université catholique de Louvain) for assistance in FACS experiments; V. Mohymont for help in real-time PCR experiments; F. Laurent and J. Josse (INSERM U1111, International Center for Research in Infectiology, Lyon, France) for providing MG63 osteoblasts cells; J.P. Pirnay and G. Verween (Queen Astrid Military Hospital, Brussels, Belgium) for providing human keratinocytes; S. Pyr dit Ruys (De Duve Institute, Université catholique de Louvain) and M. Putrinš (Institute of Technology, University of Tartu, Tartu, Estonia) for inspiring discussions, and D. Schryer for proofreading of the manuscript. This work was supported by the Belgian Fonds de la Recherche Scientifique (FNRS-FRS) (grants T.0189.16 and J.0018.17) and the Interuniversity Attraction Poles Program initiated by the Belgian Science Policy Office (program IAP P7/28). F.P. is recipient of a PhD fellowship from the Université catholique de Louvain; T.K.N. was successively junior research fellow from the Fonds de coopération 
of the Université catholique de Louvain and from the Académie de Recherche et d'Enseignement Supérieur (ARES); F.V.B. is Research Director of the Belgian FNRS-FRS; C. W. is funded by the Deutsche Forschungsgemeinschaft (SFB156); T.T. is funded by the Estonian Research Council (PRG335). The Transcriptome and Epigenome Platform is a member of the France Génomique consortium (ANR10-NBS-09-08).

\section{Author contributions}

F.P. designed and conducted the experiments, analyzed and interpreted the data. T.K.N. performed real-time PCR experiments. H.V., R.L. and O.S. performed RNA-seq and data processing. J.Y.C. supervised the RNA-seq experiments. C.W. provided key mutant strains. C.W. and T.T. contributed to data analysis and interpretation. F.V.B. supervised the work. F.P. and F.V.B. wrote the paper, which was edited and approved by the other coauthors.

\section{Competing interests}

The authors declare no competing interests.

\section{Additional information}

Supplementary information is available for this paper at https://doi.org/10.1038/s41467020-15966-7.

Correspondence and requests for materials should be addressed to F.B.
Peer review information Nature Communications thanks Lars Barquist, Konrad Förstner and the other, anonymous, reviewer for their contribution to the peer review of this work.

Reprints and permission information is available at http://www.nature.com/reprints

Publisher's note Springer Nature remains neutral with regard to jurisdictional claims in published maps and institutional affiliations.

\section{(c) (i)}

Open Access This article is licensed under a Creative Commons Attribution 4.0 International License, which permits use, sharing, adaptation, distribution and reproduction in any medium or format, as long as you give appropriate credit to the original author(s) and the source, provide a link to the Creative Commons license, and indicate if changes were made. The images or other third party material in this article are included in the article's Creative Commons license, unless indicated otherwise in a credit line to the material. If material is not included in the article's Creative Commons license and your intended use is not permitted by statutory regulation or exceeds the permitted use, you will need to obtain permission directly from the copyright holder. To view a copy of this license, visit http://creativecommons.org/ licenses/by/4.0/.

(c) The Author(s) 2020 